\title{
Time dependent fracture of soft materials: linear versus nonlinear viscoelasticity
}

\author{
Jingyi Guo, ${ }^{a}$ Alan T. Zehnder, ${ }^{a}$ Costantino Creton (D) ${ }^{\mathrm{b}}$ and Chung-Yuen Hui (D) *ac \\ Toughness of soft materials such as elastomers and gels depends on their ability to dissipate energy \\ and to reduce stress concentration at the crack tip. The primary energy dissipation mechanism is \\ viscoelasticity. Most analyses and models of fracture are based on linear viscoelastic theory (LVT) where \\ strains are assumed to be small and the relaxation mechanisms are independent of stress or strain \\ history. A well-known paradox is that the size of the dissipative zone predicted by LVT is unrealistically \\ small. Here we use a physically based nonlinear viscoelastic model to illustrate why the linear theory \\ breaks down. Using this nonlinear model and analogs of crack problems, we give a plausible resolution \\ to this paradox. In our model, viscoelasticity arises from the breaking and healing of physical cross-links \\ in the polymer network. When the deformation is small, the kinetics of bond breaking and healing are \\ independent of the strain/stress history and the model reduces to the standard linear theory. For large \\ deformations, localized bond breaking damages the material near the crack tip, reducing stress \\ concentration and dissipating energy at the same time. The damage zone size is a new length scale \\ which depends on the strain required to accelerate bond breaking kinetics. These effects are illustrated \\ by considering two cases with stress concentrations: the evolution of spherical damage in a viscoelastic \\ body subjected to internal pressure, and a zero degree peel test.
}

\section{Introduction}

Toughness of materials is due to energy dissipation and stress relaxation mechanisms at the crack tip. The toughening mechanisms of stiff materials such as metals are based on plastic deformation. Plastic flow is due to generation and motion of dislocations. It is an irreversible process that occurs when the stress exceeds the yield strength. Some elastic solids such as glass and ceramics have a limited ability to deform plastically and are brittle due to the high elastic stresses at the crack tip (in linear elastic solids, the stress has an inverse square root singularity as the crack tip is approached). In metals, yielding occurs in a region surrounding the crack tip, shielding the crack from the high elastic stress field.

The dissipative mechanism commonly associated with soft materials such as rubbers and self-healing hydrogels is viscoelasticity. ${ }^{1,2}$ Unlike metals, rubbers and gels consist of large flexible macromolecules interacting with their neighboring molecules by weak bonds. A typical flexible polymer chain in these materials is coiled and highly entangled with other polymer chains and pervades a volume of space much greater than atomic dimensions. As a result, chains are subjected to long and short range forces. It is therefore not surprising that when subjected to stress, the dynamics of chain conformation covers a wide range of time scales - the physical basis of viscoelasticity. Compared with metals, soft materials can sustain much larger strains before failure, sometimes in excess of 1000 percent. Nevertheless, in cross-linked networks, deformation is usually reversible: given enough time, the material recovers its shape after unloading to zero stress due to entropic elasticity, i.e. the coiled conformation of the molecule is energetically more favorable than any stretched conformation.

The viscoelastic model commonly used in mechanics is based on the Boltzmann superposition principle where the stress is a linear functional of the strain history. For example, in simple shear, the shear stress $\tau(t)$ at time $t$ is related to the shear strain history $\gamma\left(t^{\prime}\right), t^{\prime} \in(-\infty, t)$ by

$$
\tau(t)=\int_{-\infty}^{t} \mu\left(t-t^{\prime}\right) \frac{\mathrm{d} \gamma}{\mathrm{d} t^{\prime}} \mathrm{d} t^{\prime}
$$

where $\mu(t)$ is the shear relaxation function. The key feature of a linear viscoelastic solid is that the relaxation function is independent of stress and strain history and depends only on time. In engineering applications, the relaxation function is represented by a Prony series with a finite number of terms, 
each term being associated with a particular relaxation time. A simple way to define a nonlinear viscoelastic material is that all or some of its relaxation times depend on the history of stress and strain. Here we emphasize that it is possible to include large strain kinematics in a linear viscoelastic constitutive model. For this case, eqn (1) is no longer linear - the nonlinearity comes entirely from large strain kinematics. For example, the stress and strain history of a poly(vinylalcohol) (PVA) dual crosslinked hydrogel in uniaxial tension subjected to large stretches can be accurately predicted using a "linear" viscoelastic model of the form: $:^{3,4}$

$$
\sigma=\int_{-\infty}^{t} \mu\left(t-t^{\prime}\right) \frac{\mathrm{d}}{\mathrm{d} t^{\prime}}\left[\frac{\lambda\left(t^{\prime}\right)}{\lambda^{2}(t)}-\frac{\lambda(t)}{\lambda^{2}\left(t^{\prime}\right)}\right] \mathrm{d} t^{\prime}
$$

where $\sigma$ is the nominal stress, $\lambda$ is the stretch ratio and $\mu$ is the shear relaxation function in eqn (1). Note that eqn (2) is a nonlinear equation: the stress is not proportional to the stretch ratio. For small strains where $\lambda \approx 1$, eqn (2) reduces to eqn (1). While models of this type are often called nonlinear viscoelastic, they will be considered as linear viscoelastic in this work, since the relaxation function is independent of stress and strain history. In other words, we separate nonlinearity due to the physics of relaxation from nonlinearity caused by large strain kinematics.

Linear viscoelastic fracture (LVF) has been extensively studied starting from the seminal works of Knauss ${ }^{5}$ and Schapery ${ }^{6}$ in the seventies. More recent contributions include works by de Gennes, Hui, Persson and Nguyen. ${ }^{7-10}$ More references can be found in a recent review by Knauss. ${ }^{11}$ There are two well-known paradoxes associated with LVF. For a growing crack, the extended correspondence principle of Graham ${ }^{12}$ showed that the stress field at the crack tip has the same square root singularity as the elastic crack problem. This result implies that there is a local energy release rate at the moving crack tip. However, the absence of a length scale in the linear viscoelastic model results in a local energy release rate that is independent of crack speed - a paradox. An excellent summary of this paradox can be found in Rice. ${ }^{13}$ Readers interested in history are encouraged to read the spirited discussions of McCartney and Christiansen. ${ }^{14,15}$ This paradox was resolved by Knauss ${ }^{5}$ and Schapery ${ }^{6}$ who used a cohesive zone model (CZM) to remove the stress singularity. The CZM also introduces a length scale which can be identified as the size of the dissipative zone. The CZM, however, creates a different paradox as noted by the works of Mueller ${ }^{16}$ and Gent. ${ }^{2}$ For example, Gent and Lai $^{2}$ interpreted their peel test data of rubber using Knauss's theory and using the linear viscoelastic properties of their material to find that the size of the dissipation zone was only on the order of angstroms. Finite element simulations of a peel test using a linear viscoelastic model with large strain kinematics by Rahulkumar et $a .^{17}$ also support this conclusion. More evidence of the very small size of the cohesive zone is summarized in a recent review by Knauss. ${ }^{11}$ Thus, this implies rather unphysically that a huge amount of energy is dissipated in a very small volume. Gent ${ }^{2}$ suggested intermittent crack growth as a plausible explanation for this paradox. Knauss ${ }^{18}$ suggested nonlinear viscoelasticity caused by dilation induced softening of the material near the crack tip.
Indeed, the strains/stresses near the crack tip are so large that it is unlikely that the material near the crack tip can be adequately described by linear viscoelasticity. As we shall demonstrate below, nonlinear viscoelasticity introduces a length scale at the crack tip which can be related to the size of the dissipative zone.

As noted by Knauss, ${ }^{11}$ most studies on nonlinear viscoelasticity constitutive models are one dimensional in character and cannot be used to study the complex multi-axial stress state near the crack tip. Also, there are very few attempts to study fracture in soft materials where large deformation is coupled with nonlinear viscoelasticity. Indeed, linear viscoelasticity works well only when the strains are small. In soft solids, the nominal strains can easily exceed several hundred percent in a simple tension test, hence linear theory is not expected to work near the crack tip.

Our way of highlighting the different physics associated with linear and nonlinear viscoelasticity is to consider a polymer network connected by chemical and physical cross-links. ${ }^{19}$ In the past decade, gels made of this type of network have been widely studied for their useful properties such as high toughness and self-healing capability. ${ }^{19-23}$ The chemical cross-links in these networks are covalent bonds, and their failure is rate insensitive: they fail if they are stretched beyond a certain limit. The physical cross-links are weaker bonds with a distribution in strength. They can break and reattach or heal. For this type of network, viscoelasticity is associated with the bond breaking and healing kinetics. Linear viscoelasticity results when the bond breaking and healing kinetics are independent of the stretch on the chains and the breaking and healing process has achieved dynamic equilibrium. For this case there is a one to one correspondence between the relaxation function and the stress/strain independent breaking kinetics ${ }^{19}$ (see also Section 4.1). An example of a linear viscoelastic gel is the polyvinyl-alcohol (PVA) dual crosslinked gel with PVA chemically crosslinked by glutaraldehyde and physically crosslinked by Borax ions. ${ }^{19,24}$ Experiments have demonstrated that the (probably complex) bond breaking kinetics of this gel is insensitive to the stress acting on the physical cross-links. Here we note that the use of a transient network to model viscoelasticity in solids has been known for a long time, starting with the seminal work of Green and Tobolsky. ${ }^{25}$ Since then, many researchers have used transient networks to model the time dependent behavior of rubbers and gels. ${ }^{26,27}$ How the dynamics of physical bonds controls the linear viscoelastic behavior of polymeric fluids has also been very well studied both theoretically ${ }^{28-30}$ and experimentally. ${ }^{31,32}$

However, molecular physics tells us that bond breaking and healing kinetics should be sensitive to stress/strain. Indeed, linear viscoelasticity is counter-intuitive for this case since Eyring's theory states that bonds dissociate faster when they are stretched. ${ }^{33}$ It is therefore not surprising that linear viscoelastic theory typically works well in the regime of small strains where the effect of stress on bond breaking can be neglected. However, if the dynamics of bond breaking and healing are sensitive to stress/strain especially when deformation is large, then it is reasonable to expect the material behavior near the crack tip cannot be adequately described by linear viscoelasticity. 
Of course, there are physical mechanisms other than breaking of physical cross-links that can lead to nonlinear viscoelasticity; indeed, our analysis does not rely on this physical assumption. This assumption merely lends a physical picture on how nonlinear viscoelasticity differs from the linear theory.

The plan of this paper is as follows. Section 2 summarizes some aspects of LVF theory relevant to this work. In Sections 3 and 4 we contrast linear and nonlinear viscoelasticity using a physically based model of chain breaking and healing kinetics. We highlight the differences between these two models by showing that nonlinear viscoelasticity can lead to local damage or softening, and that such damage shields the crack tip from the high elastic stress field. The size of the damage zone is a new length scale. In Section 4 we introduce a special nonlinear viscoelasticity model and use it to study two problems with stress concentrations: the inflation of a spherical cavity subjected to internal pressure, and a zero degree peel test. We compare the solutions of these two problems based on nonlinear and linear theory. Here we emphasize that our goal is not to study the physics of cavitation and adhesion - work on these topics can be found in ref. 34 and 35 and the references within. Our goal is to use these as examples of stress concentrations to contrast nonlinear and linear viscoelastic behaviors. Discussion and summary are given in Section 5 .

\section{Brief review of linear viscoelastic fracture (LVF) theory}

We indicate certain peculiarities of LVT theory using examples. Since many soft materials are close to being incompressible, we simplify the mathematics by assuming that the material is incompressible so that its mechanical behavior can be described by a single shear relaxation function. A key feature of linear viscoelasticity is the correspondence principle which allows one to convert static viscoelastic solutions to elastic solutions. ${ }^{36}$ A simple example is a stationary traction free plane stress or plane strain crack in a linear viscoelastic body subjected to traction boundary conditions. For this case, the stresses in the viscoelastic body are exactly the same as the stresses in an elastic body with identical geometry and subjected to the same traction, in particular, they are independent of the elastic modulus. For example, consider a finite plane stress/plane strain crack of length $2 a$ in an infinite linear viscoelastic solid. The body is initially stress free; at time $t=0^{+}$a constant tension $\sigma_{22}\left(x_{1}, x_{2}= \pm \infty, t>0\right)=\sigma_{\infty}$ is imposed at infinity. The correspondence principle states that the stress remains independent of time for $t>0$ and is given by the elastic solution. With respect to a polar coordinate system $(r, \theta)$ with origin at the crack tip, the in-plane crack tip stresses are given by

$$
\sigma_{\alpha \beta}(r \rightarrow 0, \theta)=\frac{K_{\mathrm{I}}}{\sqrt{2 \pi r}} \hat{\sigma}_{\alpha \beta}(\theta) \quad \alpha, \beta=1,2
$$

where $K_{\mathrm{I}}=\sigma_{\infty} \sqrt{\pi a}$ is the Mode I stress intensity factor and $\hat{\sigma}_{\alpha \beta}(\theta)$ are universal functions that describe the angular variation of the stress components. ${ }^{37}$ Thus, in problems where traction is prescribed, viscoelastic flow does not shield the crack tip from the high stress of the elastic solution. Further, the strains will increase with time according to the creep function. As a result, viscoelasticity increases the strain and does nothing to alleviate the stress field near the crack tip. This in turn should not protect chemical bonds at the crack tip from failure.

\section{Nonlinear viscoelasticity}

We construct a nonlinear viscoelastic solid by removing the assumption that the physical cross-links can support arbitrarily large stresses/strains without increasing their breaking rate. In this nonlinear theory, the breaking kinetics depend on the stretch of the chains between physical cross-links. Before we dive into the details, let's examine the consequence of this new theory. Let us suppose that the rate of breaking of physical bonds increases rapidly when a chain between two physical cross-links is stretched beyond a critical level. This means that near the crack tip, practically all the physical cross-links are not carrying load. Thus the material near the crack tip relaxes much faster than the material away from the crack tip. For concreteness, let's compare two identical crack geometries A and B. Both $\mathrm{A}$ and $\mathrm{B}$ are subjected to identical traction boundary conditions, for example, a finite crack in an infinite plate subjected to a constant remote tension $\sigma_{\infty}$ for time $t>0^{+}$. The material occupying A is linear viscoelastic and the material occupying B is nonlinear viscoelastic. We assume both materials have the same long and short time shear moduli. Before load is applied, $t<0$, the physical cross-links in A and B are in dynamic equilibrium and the short time modulus $\mu_{0}$ is determined by the number of load bearing chemical and physical cross-links. The long time modulus, $\mu_{\infty}$, is by definition, the modulus when all the physical cross-links are broken. In A, the stresses near the crack tip are given by eqn (3), with $K_{\mathrm{IA}}=\sigma_{\infty} \sqrt{\pi a}$, independent of material properties. However, in $\mathrm{B}$, there is a region $\Omega$ surrounding the crack tip where relaxation occurs much faster - now material behavior is spatially inhomogeneous. The consequence of this stress induced softening can be studied using the simple model illustrated in Fig. 1. Here we assume $\Omega$ is a circle of radius $R$ centered at the crack tip. Further, we assume that breaking of the physical cross-links occurs so fast that the material inside $\Omega$ behaves like an elastic solid whose elasticity is controlled by the chemical crosslinks, with the relaxed or plateau modulus $\mu_{\infty}$. Outside $\Omega$ the material has a shear modulus $\mu \gg \mu_{\infty}$. Since the material inside $\Omega$ is linear elastic, the stress field near the crack tip in B still has the same form given by eqn (3), but the stress intensity factor $K_{\mathrm{IB}}$ is expected to be much smaller than $K_{\mathrm{IA}}$ (note $K_{\mathrm{IB}} \rightarrow 0$ as $\mu_{\infty} \rightarrow 0$ (a hole)) since most of the load is shed to the stiffer region outside $\Omega$ : the material near the crack tip is shielded from the high stress field of specimen A.

To understand this shielding effect more quantitatively consider a simple problem, that of a semi-infinite Mode III crack in an infinite block of an elastic solid, as sketched in Fig. 2. The damage zone (where most of the physical cross-links are broken) is denoted 
(a)

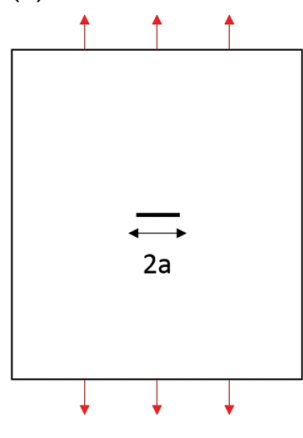

A (b)

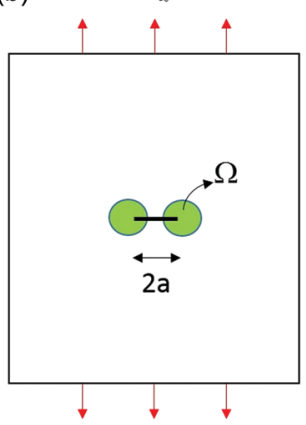

B

Fig. 1 A finite plane stress or plane strain crack of length $2 a$ in an infinite body. Geometries of $A$ and $B$ are identical. $A$ is linear viscoelastic (a) and $B$ is nonlinear viscoelastic (b). Both A and B have the same long and short time shear moduli and the same small strain response. There is no damage zone in (a) in the sense that viscoelastic properties are the same everywhere (the physical cross-links can reform and bear load). In (b), there is a damage zone (inside the green circle) where most of the physical bonds are not bearing load.

by $\Omega$. Inside $\Omega$, the material is linear elastic with a lower shear modulus $\mu_{\infty}$. The material outside the inclusion has shear modulus $\mu \gg \mu_{\infty}$. The boundary condition in the far field is

$$
\sigma_{r z}(r \rightarrow \infty, \theta)=\frac{K_{\text {III }}}{\sqrt{2 \pi r}} \sin (\theta / 2)
$$

This elasticity problem was solved by Steif, ${ }^{38}$ the stress intensity factor $K_{\mathrm{III}}^{\mathrm{in}}$ inside the soft inclusion is reduced by a factor of $\frac{2 \mu_{\infty}}{\mu+\mu_{\infty}} \ll 1$, i.e.,

$$
K_{\mathrm{III}}^{\text {in }}=\frac{2 \mu_{\infty}}{\mu+\mu_{\infty}} K_{\text {III }}
$$

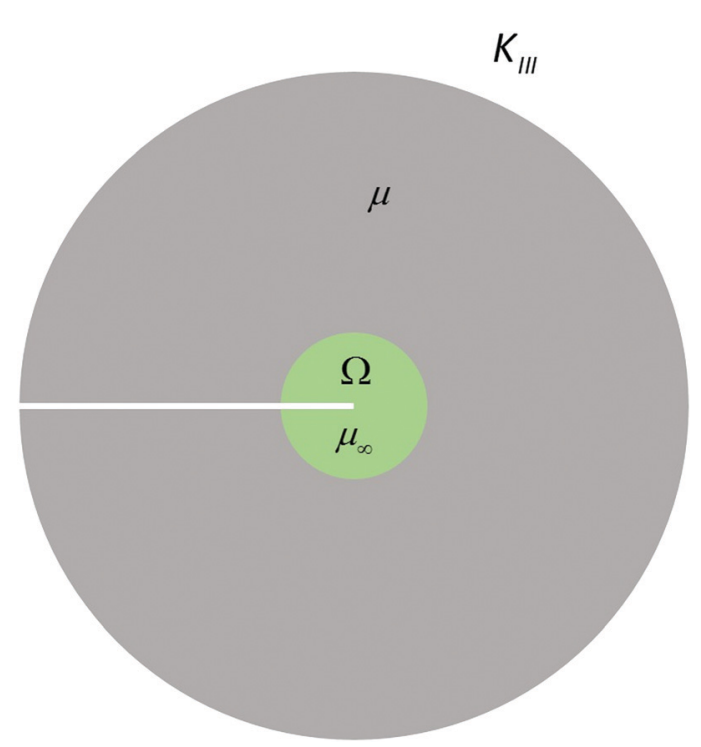

Fig. 2 Semi-infinite Mode III crack subjected to a remote applied $K_{\text {III }}$ field. The damage zone is denoted by $\Omega$ and has shear modulus $\mu_{\infty} \ll \mu$, where $\mu$ is the shear modulus of the undamaged material outside.

The external applied energy release rate $\Gamma_{\text {ext }}$ is

$$
\Gamma_{\text {ext }}=\frac{K_{\mathrm{III}}^{2}}{2 \mu}
$$

whereas the local energy release rate $\Gamma_{\text {local }}$ is

$$
\Gamma_{\text {local }}=\frac{\left(K_{\text {III }}^{\text {in }}\right)^{2}}{2 \mu_{\infty}}=\left(\frac{2 \mu_{\infty}}{\mu+\mu_{\infty}}\right)^{2} \frac{K_{\text {III }}^{2}}{2 \mu_{\infty}}
$$

The ratio of the energy release rates is obtained by combining (6a) and (6b),

$$
\frac{\Gamma_{\text {local }}}{\Gamma_{\mathrm{ext}}}=\left(\frac{2 \mu_{\infty}}{\mu+\mu_{\infty}}\right) \frac{2 \mu}{\mu+\mu_{\infty}} \approx \frac{4 \mu_{\infty}}{\mu+\mu_{\infty}}
$$

Thus, the local energy release rate is also reduced; indeed, by almost twice the factor of reduction of the local stress intensity factor. This reduction in energy release rate is due to the fact that energy is dissipated by the rapid breaking of physical crosslinks due to the transition from $\mu \rightarrow \mu_{\infty}$ in $\Omega$. Note that an analysis for a Mode I crack was also carried out by Steif, ${ }^{38}$ yielding similar results. We use the Mode III crack as an example since the analytical expressions have simpler forms. In addition, our focus is on explaining the fundamental physics that distinguishes linear and nonlinear viscoelasticity in stress concentration problems, so examples and analogies can be made across different modes of fracture.

Thus, our nonlinear viscoelastic model identifies a length scale (the size of $\Omega$ ) where material damage and rapid stress relaxation can occur. Note that the correspondence principle does not hold since the material property is no longer independent of stress. Further, if the crack grows, one would expect that the size of the damage zone as well as the strain and stress field inside it will depend on the crack speed; hence there is no need to use a cohesive zone model to introduce a length scale even though the crack tip stress can still be unbounded. This is not surprising, the strain fields of growing cracks in elasticplastic solids can have a weak singularity inside the plastic zone. ${ }^{39}$ The size of this damage region is directly related to the size of the dissipative zone: at this point we are unable to determine the size of this zone; to do so we need to use a nonlinear viscoelastic model, which is discussed below.

The above example is clearly over-simplified since in reality the transition from stiff to soft or the amount of softening is a smooth function of position. In particular, $\Omega$ does not need to be a circle. Furthermore, the material outside of $\Omega$ is not elastic, since it can also relax with time, though at a slower rate. In addition, we also assume small strains which is not usually the case for soft materials. Nevertheless, it supports our idea that:

1. Nonlinear viscoelasticity shields the crack from the high stress field predicted by the linear theory, and reduces the local energy rate available for fracture.

2. Local damage due to rapid bond dissociation gives rise to a new length scale which can be related to the size of the dissipation zone. 
In the following, we will introduce a specific nonlinear viscoelasticity model and use it as an example to quantify these ideas for two problems.

\section{Two stress concentration problems: inflation of a spherical cavity by internal pressure and the zero degree peel test}

Ideally, we should compare the time dependent large deformation stress and strain fields near the tip of a crack for linear and nonlinear models. However, this is a very difficult numerical problem for the nonlinear model since the entire history of deformation at every material point needs to be stored and the memory requirement is exceedingly large. We avoid this difficulty by considering two well-known stress concentration problems as analogs: The first is a spherical cavity in an infinite viscoelastic solid subjected to an internal pressure $p>0$. The second is a zero degree peel test. Here we note that the spherical cavity problem has been studied using both nonlinear elastic $^{40,41}$ and viscoelastic models. ${ }^{42,43}$ Our focus here is not on cavitation phenomena but on highlighting the stress shielding effect of nonlinear viscoelasticity based on the model introduced below.

\subsection{Nonlinear viscoelastic model}

In a series of papers, we have demonstrated that the mechanical behavior of a dual cross-linked PVA hydrogel can be modeled very well by a linear viscoelastic model provided that we account for large deformation. ${ }^{3,44,45}$ Here we extend this linear model to a nonlinear model by allowing the bond breaking kinetics to be dependent on the strain history. To compare the two theories, it is necessary that both linear and nonlinear model have the same short and long time relaxation moduli; in addition, the nonlinear model should reduce to the linear model in the limit of small strains. These conditions are imposed on the examples in Sections 4.2 and 4.3.

4.1.1 Review of the linear model. First, we briefly summarize our linear viscoelastic model, details can be found in ref. 3 and 44. Let $\rho$ denote the molar fraction of chemical crosslinks and $W_{0}$ the strain energy per unit volume in the undamaged network. We assume $W_{0}$ to be the same for both physical and chemical network. For simplicity, we assume $W_{0}$ to be the neoHookean strain energy density function, i.e.,

$$
W_{0}=\frac{\mu_{\text {net }}}{2}\left(I_{1}-3\right)
$$

where $I_{1}$ is the trace of right Cauchy-Green tensor and $\mu_{\text {net }}$ is the modulus of the network if all the bonds bear load. We assume the breaking and healing process has achieved dynamic equilibrium soon after the gel is made. Thus, the healing rate is equal to the breaking rate and is denoted by the rate constant $\chi_{\infty}$. The breaking kinetics of physical crosslinks is specified by the function $\phi_{\mathrm{B}}\left(t^{\prime}, t\right)$ which is defined as the fraction of chains per unit reference volume which reattaches at $\tau$ and survives till $t \geq t^{\prime}$, where $t$ is the current time. Physically, $\phi_{\mathrm{B}}\left(t^{\prime}, t\right)$ is the survival probability of physical chains and its value at $t=t^{\prime}$ is 1 and at $t=\infty$ is zero. In the linear theory, $\phi_{\mathrm{B}}\left(t^{\prime}, t\right)$ is independent of the stretch experienced by the physical chains. For this case, $\phi_{\mathrm{B}}\left(t^{\prime}, t\right)$ is translational invariant, that is, $\phi_{\mathrm{B}}\left(t^{\prime}, t\right)=\phi_{\mathrm{B}}\left(t-t^{\prime}\right)$. More importantly, the shear relaxation function $\mu(t)$ is completely determined by $\phi_{\mathrm{B}}$ through the simple relation:

$$
\frac{\mathrm{d} \mu}{\mathrm{d} t}=-\chi_{\infty} \mu_{\mathrm{net}} \phi_{\mathrm{B}}(t),
$$

where $\phi_{\mathrm{B}}(t)=\phi_{\mathrm{B}}(\tau=0, t)$. Eqn (8) states that there is a one-to-one correspondence between linear viscoelasticity and chain breaking kinetics, so one can generate any relaxation function by specifying the appropriate survivability function. In our linear viscoelastic model for the PVA gel, the survival probability function $\phi_{\mathrm{B}}(t)$ is

$$
\phi_{\mathrm{B}}(t)=\left[1+\left(\alpha_{\mathrm{B}}-1\right) \frac{t}{t_{\mathrm{B}}}\right]^{\frac{-1}{\alpha_{\mathrm{B}}-1}}
$$

where $\alpha_{\mathrm{B}}$ is a material constant satisfying $2>\alpha_{\mathrm{B}}>1$ and $t_{\mathrm{B}}$ is the characteristic time of breaking of physical crosslinks. The shear relaxation function $\mu(t)$ in this case is obtained using eqn (8) and (9) and is

$$
\mu(t)=\mu_{\text {net }} \rho+\mu_{\text {net }} \chi_{\infty} \frac{t_{\mathrm{B}}}{2-\alpha_{\mathrm{B}}}\left[\phi_{\mathrm{B}}(t)\right]^{2-\alpha_{\mathrm{B}}} .
$$

The instantaneous modulus is

$$
\mu_{0} \equiv \mu(t=0)=\mu_{\text {net }} \rho+\mu_{\text {net }} \chi_{\infty} \frac{t_{\mathrm{B}}}{2-\alpha_{\mathrm{B}}} .
$$

The long time modulus is

$$
\mu_{\infty} \equiv \mu(t \rightarrow \infty)=\mu_{\text {net }} \rho .
$$

We emphasize that in the linear theory the survival probability is independent of the stress or strain acting on a chain. For large deformation, the true stress tensor $\sigma$ is related to the deformation history by:

$$
\boldsymbol{\sigma}=-q \mathbf{I}+\mu_{\text {net }} \rho \mathbf{B}^{0 \rightarrow t}+\mu_{\text {net }} \chi_{\infty} \int_{-\infty}^{t} \phi_{\mathrm{B}}\left(t-t^{\prime}\right) \mathbf{B}^{t^{\prime} \rightarrow t} \mathrm{~d} t^{\prime}
$$

where $q$ is the Lagrange multiplier that enforces incompressibility, I is the identity tensor, and $\mathbf{B}=\mathbf{F F}^{\mathrm{T}}$ is the left Cauchy-Green tensor. Here the superscript $t^{\prime} \rightarrow t$ in $\mathbf{B}^{t^{\prime} \rightarrow t}$ indicates the deformation experienced by physical chains reattached at time $t^{\prime}<t$ and survive till $t$.

4.1.2 A nonlinear model. We now extend the linear model (11) to a nonlinear model (details are given in the appendix) by allowing the breaking rate to be strain dependent. Specifically, the survivability function $\phi_{\mathrm{B}}\left(t^{\prime}, t\right)$ is no longer independent of the strain history. Instead of eqn (9), it is determined by

$$
\phi_{\mathrm{B}}\left(t^{\prime}, t\right)=\left\{1+\frac{\alpha_{\mathrm{B}}-1}{t_{\mathrm{B}}} \int_{t^{\prime}}^{t}\left[1+\frac{H\left(t^{\prime}, s\right)-3}{J_{0}^{2}}\right]^{m} \mathrm{~d} s\right\}^{\frac{-1}{\alpha_{\mathrm{B}}-1}}
$$

where $H\left(t^{\prime}, t\right) \equiv \operatorname{tr}\left[\left(\mathbf{F}^{t^{\prime} \rightarrow t}\right)^{\mathrm{T}} \mathbf{F}^{t^{\prime} \rightarrow t}\right]$. Physically, $H\left(t^{\prime}, t\right)$ measures the square of the effective stretch of chains from the time they 
are born $t^{\prime}$ to the current time $t$. The dimensionless constant $J_{0}$ can be viewed as a critical strain above which breaking rate increase rapidly, and $m>0$ is a material constant that describes the breaking rate's dependence on the deformation. In particular, when deformation is small, or equivalently, $\left(H\left(t^{\prime}, s\right)-3\right) / J_{0}^{2} \ll 1$, eqn (12) reduces to eqn (9). Hence the nonlinear theory reduces to the linear theory in the limit of small strains. Clearly, the instantaneous and long time moduli are the same for both linear and nonlinear model.

A consequence of strain dependent bond breaking is that dynamic equilibrium cannot be achieved when the material is under load, so $\chi_{\infty}$ in eqn (11) must be replaced by a history dependent healing rate which satisfies an integral equation. We denote the healing rate at any time $t$ by $\chi(t)$, but it is important to note that $\chi(t)$ is not only a function of the current time $t$, but also depends on the entire loading history. The integral equation for $\chi(t)$ is derived in the appendix. In this nonlinear theory, eqn(11) is replaced by

$$
\boldsymbol{\sigma}=-q \mathbf{I}+\mu_{\text {net }} \rho \mathbf{B}^{0 \rightarrow t}+\mu_{\text {net }} \int_{-\infty}^{t} \chi\left(t^{\prime}\right) \phi_{\mathrm{B}}\left(t^{\prime}, t\right) \mathbf{B}^{t^{\prime} \rightarrow t} \mathrm{~d} t^{\prime}
$$

Note that when $J_{0} \rightarrow \infty$ (or equivalently, at small strains) in eqn (12), the bond breaking kinetics is independent of the history of chain stretch and we recover the linear viscoelastic model eqn (9)-(11). We emphasize that both the linear and nonlinear model have the same long time and short time moduli. Furthermore, both models allow for large deformation. Also, they have identical small strain rheological behaviors (such as loss and storage moduli) since $\left[1+\left(H\left(t^{\prime}, s\right)-3\right) / J_{0}^{2}\right]^{m} \approx 1$ in this limit.

We use a constant stretch rate uniaxial tension test to demonstrate the difference between these two models. Fig. 3 compares the true stresses of this nonlinear viscoelastic model (determined by eqn (12) and (13)) with the linear viscoelastic model using the material parameters:

$$
\rho=0.03, \alpha_{\mathrm{B}}=1.8, t_{\mathrm{B}} / t_{\mathrm{H}}=4, J_{0}=0.1, m=1.7 .
$$

where $\rho, \alpha_{\mathrm{B}}$ and $t_{\mathrm{B}} / t_{\mathrm{H}}$ are the parameters of the linear model and $J_{0}$ and $m$ are the additional parameters of the nonlinear theory. These parameters are chosen to emulate experimental data by Sun $e t a l{ }^{23}$ on a polyampholyte gel. In uniaxial tension test, the stress versus strain curves of this gel loaded at different stretch rates abruptly change slope at a critical strain that is approximately independent of the strain rate. Sun $e t a l^{23}$ refer this critical strain as the yield strain. Here we use our nonlinear model to approximately capture this behavior. In Fig. 3, the two models are identical when the stretches are small compared with the effective critical strain $J_{0}$. For large stretches, the stress/strain dependent breaking kinetics significantly softens the material. The nonlinear viscoelastic solid exhibits "history dependent yield" behavior where there is a rapid change in slope of the stress-stain curve, consistent with experiment. In our model this "yield" behavior is associated with the rapid breaking of physical cross-links when the effective strain $\sqrt{H-3}$ in eqn (12) is close to $J_{0}$. Note that for different loading rates yield occurs at strains between 0.05 and 0.1 , which is near $J_{0}$.

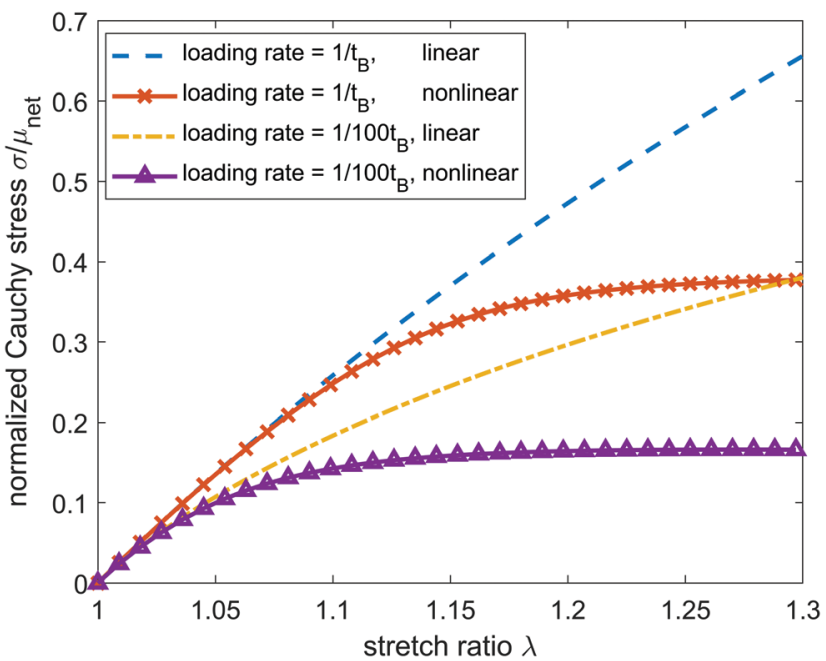

Fig. 3 Uniaxial tension test with constant loading rates $1 / t_{\mathrm{B}}$ and $1 / 100 t_{\mathrm{B}}$ for linear and nonlinear viscoelastic models.

In the experiment of Sun $e t a l .{ }^{23}$ the yield strains at different strain rates are closer than our model. A very rough estimate of the stress carried by the chains at "yield" is $\approx \mu_{\text {net }} J_{0}$. Hence we can identify a characteristic "yield stress" in shear by

$$
\sigma_{\mathrm{Y}} \approx \mu_{\text {net }} J_{0} .
$$

This is an upper estimate since $\mu_{\text {net }}$ is the shear modulus when all the chains are bearing load. As shown in Fig. 3, the "yield stress" is rate dependent since at slower rates, the physical bonds can break and the effective shear modulus goes down.

\subsection{Inflation of a cavity in a linear viscoelastic/nonlinear viscoelastic solid}

The first case we study is the inflation of a spherical cavity in an infinite viscoelastic solid subjected to an internal pressure $p>0$, Fig. 4. The stress free reference configuration is a traction free cavity with initial undeformed radius is $A$. In the following, the deformed radius of the sphere is denoted by $a$. As we shall see, this problem bears some similarity to a crack problem, in the sense that the hoop stress on the surface of the cavity becomes infinite at some critical pressure.

First, recall that a cavity in an incompressible neo-Hookean body will become unstable and grow without bound when the internal pressure $p$ reaches the critical value of $p_{\mathrm{c}}=5 \mu_{0} / 2$, where $\mu_{0}$ is the small strain shear modulus. ${ }^{46,47}$ In particular, the true hoop stress goes to infinity at $p_{\mathrm{c}}=5 \mu_{0} / 2$. Let $\sigma_{\theta}$ denote the true hoop stress and define a stress concentration factor

$$
k \equiv \max \left(\sigma_{\theta} / p\right)
$$

The maximum true hoop stress occurs on the surface of cavity and is

$$
\sigma_{\theta}^{\max }=\mu_{0}\left(\bar{a}^{2}-\frac{1}{2 \bar{a}^{4}}+\frac{2}{\bar{a}}-\frac{5}{2}\right)
$$




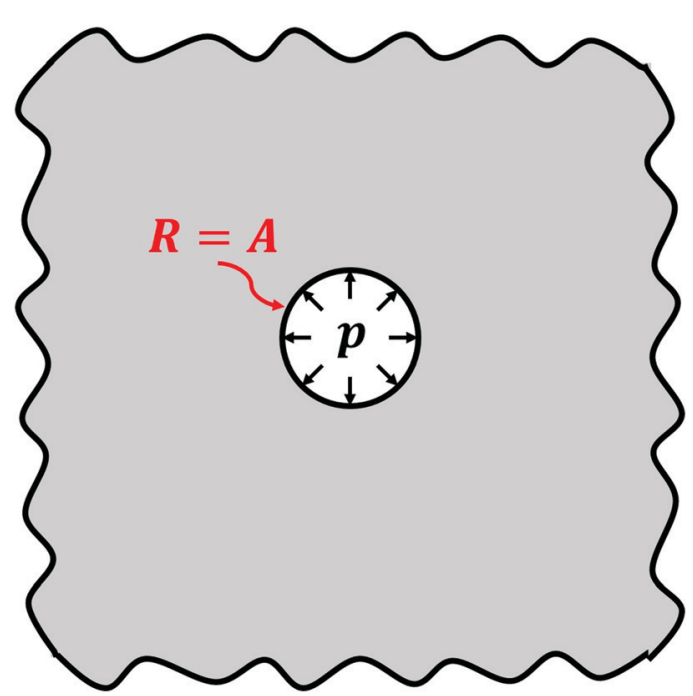

Fig. 4 Schematic of an infinite body with a spherical hole inside. An internal pressure of $p$ is applied to the spherical cavity. The cavity has initial radius $A$.

where $\bar{a}=a / A$ is the normalized deformed radius and is related to the applied pressure $p$ by

$$
\frac{1}{\bar{a}^{4}}+\frac{4}{\bar{a}}=5-\frac{2 p}{\mu_{0}}
$$
Eqn (17b) implies that, as $p \rightarrow 5 \mu_{0} / 2, \bar{a} \approx \frac{2 \mu_{0}}{\frac{5 \mu_{0}}{2}-p} \rightarrow \infty$ and
by eqn (17a),

$$
\sigma_{\theta}^{\max } \rightarrow \mu_{0} \bar{a}^{2} \approx \frac{4 \mu_{0}^{3}}{\left(\frac{5 \mu_{0}}{2}-p\right)^{2}} \rightarrow \infty
$$

Note that (18) is valid only for large strains. Here it is interesting to recall that in small strain theory, the stress concentration factor is exactly $1 / 2 .{ }^{48}$ As deformation becomes large, the stress concentration factor increases rapidly and approaches infinity when $p$ approaches $5 \mu_{0} / 2$. This result shows that large errors can occur using small strain theory in large deformation problems. The infinitely large tensile hoop stress near the cavity surface predicted by eqn (18) suggests that small defects can grow into macroscopic cracks before instability can occur.

4.2.1 Inflation of cavity in a linear viscoelastic solid. Next, we replace the neo-Hookean solid by the linear viscoelastic model described in Section 4.1.1 (the strains can still be arbitrary large). The geometry is the same as Fig. 4 . At time $t=0^{+}$, a sudden pressure $p$ is imposed on the cavity surface. This pressure is held constant for all $t>0$. In the appendix, we show that the deformed radius $a(t)$ satisfies the integral equation

$$
\mu(t)\left[\frac{4}{\bar{a}(t)}+\frac{1}{\bar{a}^{4}(t)}\right]+\int_{0}^{t} \frac{\partial \mu\left(t-t^{\prime}\right)}{\partial \tau}\left[\frac{4 \bar{a}\left(t^{\prime}\right)}{\bar{a}(t)}+\frac{\bar{a}^{4}\left(t^{\prime}\right)}{\bar{a}^{4}(t)}\right] \mathrm{d} t^{\prime}=-2 p+5 \mu_{0},
$$

where $\bar{a}=a(t) / A$ is the normalized current radius and $\mu(t)$ is the relaxation function given by eqn (10a). The maximum hoop stress for this case also occurs on the cavity surface and is related to the history of cavity growth by

$$
\begin{aligned}
\sigma_{\theta}^{\max }= & \mu(t)\left[\frac{2}{\bar{a}(t)}-\frac{1}{2 \bar{a}^{4}(t)}+\bar{a}^{2}(t)\right] \\
& -\frac{5}{2} \mu_{0}+\int_{0}^{t} \frac{\partial \mu\left(t-t^{\prime}\right)}{\partial t^{\prime}}\left[\frac{2 \bar{a}\left(t^{\prime}\right)}{\bar{a}(t)}-\frac{\bar{a}^{4}\left(t^{\prime}\right)}{2 \bar{a}^{4}(t)}+\frac{\bar{a}^{2}(t)}{\bar{a}^{2}\left(t^{\prime}\right)}\right] \mathrm{d} t^{\prime}
\end{aligned}
$$

Note that if the relaxation function $\mu(t)$ is a constant, then eqn (20) reduces to the case of a neo-Hookean solid. The linear viscoelastic case is easy to understand since the material is spatially homogeneous - at any instant in time, the material behaves like a homogenous neo-Hookean solid with shear modulus $\mu(t)$ where $\mu_{0} \leq \mu(t) \leq \mu_{\infty}$. Since our goal is not to study instability, the imposed pressure must be smaller than $5 \mu_{\infty} / 2$ if the cavity were to remain stable for all times, where $\mu_{\infty}$ is the long time shear modulus. Fig. $5 \mathrm{a}$ and $\mathrm{b}$ show the normalized cavity radius and the stress concentration factor $k$ versus normalized time $\bar{t} \equiv t / t_{\mathrm{B}}$ for an imposed pressure of $p=0.3 \mu_{0}$. These results are generated using the material

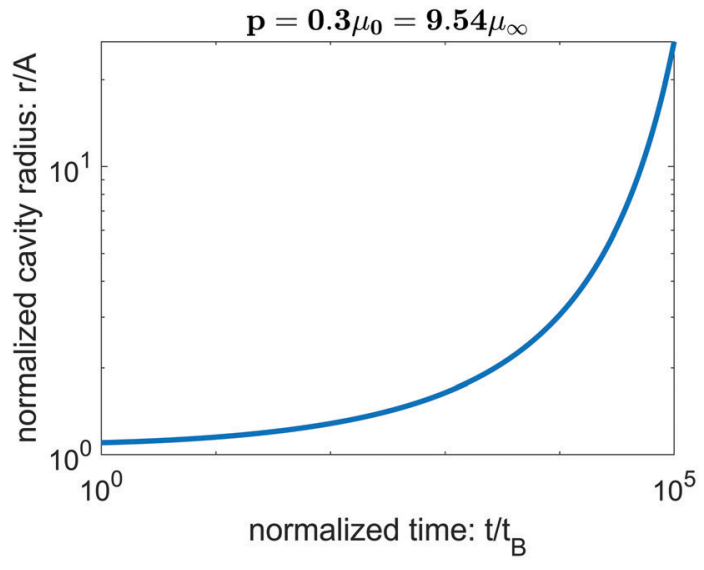

(a)

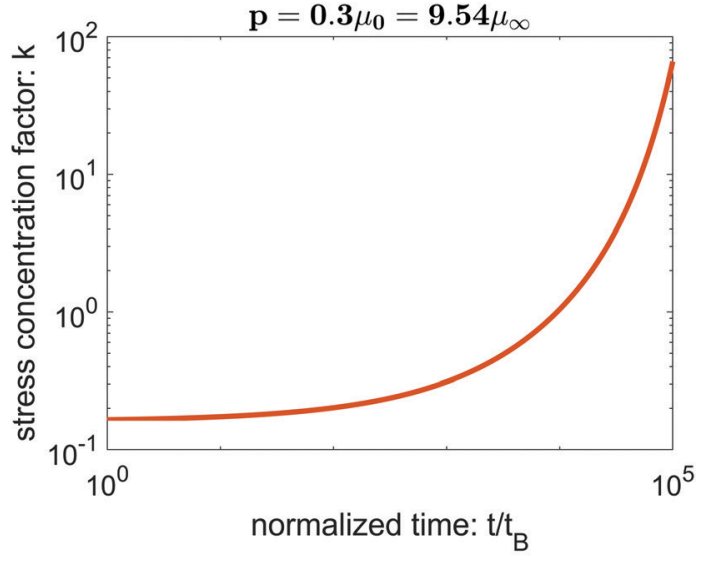

(b)

Fig. 5 Inflation of cavity in a linear viscoelastic solid: (a) normalized cavity radius and (b) Hoop stress concentration factor as a function of time. 
properties listed in eqn (14) with $\mu_{0}=31.8 \mu_{\infty}$ or $p=9.54 \mu_{\infty}$. Since $p>5 \mu_{\infty} / 2$, the cavity will grow without bound at some finite time. Our choice of parameters is such that the cavity growth rate starts to increase rapidly around $t=10^{4} t_{\mathrm{B}}$. At this time, Fig. 5b shows that the stress concentration factor increases rapidly and eventually becomes unbounded as the solid relaxes.

4.2.2 Inflation of cavity in a non-linear viscoelastic solid: shielding due to strain dependent bond breaking. We next consider the same geometry and loading condition as in the linear viscoelastic case, except the solid is now nonlinearly viscoelastic, with behavior given by eqn (13). This case is much more complex, since there exists a time dependent shell region near the cavity where the material relaxes much faster (due to rapid bond breaking near the critical pressure). This "soft" region is surrounded by a "stiffer" material. Therefore, stress will be shed to this stiff region, causing a reduction in hoop stress on the cavity surface compared with the linear case. Unfortunately, there is no closed form solution and the problem must be solved numerically. Details are given in the appendix. Fig. 6a-c compare the distribution of hoop stress at three different normalized times. The simulations are carried out using the same material parameters (same long time and short time modulus and small strain behavior) for the linear and nonlinear models and the numerical values are given in eqn (14). The pressure in these simulations is $p / \mu_{0}=0.3$. The nonlinear viscoelastic solid shows two interesting features: the hoop stress near the surface of the cavity is much lower than the corresponding linear viscoelastic solid (recall that they have the same long and short time relaxation modulus and identical small strain behavior). Much more surprising is that the hoop stress of the nonlinear case keeps decreasing with time and eventually turns compressive (see Fig. 6c). This suggests that nucleation of defects on the surface of the cavity, if it occurs, could be suppressed by shielding. It should be noted that cavity growth is faster for the nonlinear viscoelastic solid (see Fig. 11 in the appendix) because stress shielding requires that the extra load be shed to a larger region. In other words, nonlinear viscoelasticity reduces stress concentration by spreading deformation to a larger region. This is similar to the toughening mechanism of double-network gels which reduce stress concentration by developing a damage zone surrounded by a region of undamaged material.

Eqn (12) and the definition of $J_{0}$ suggests a way to define the size of damage zone. We expect that everywhere inside the damage zone the effective strain $\sqrt{I_{1}(R, t)-3}$ is greater than the critical effective strain $J_{0}$. Thus, the radius of the outer boundary of the damage zone, $R_{\mathrm{d}}$ is defined by $\sqrt{I_{1}\left(R_{\mathrm{d}}, t\right)-3}=J_{0}$. The size of the damage zone $D(t)$ is defined as the thickness of this shell which is $D \equiv R_{\mathrm{d}}-A$. Fig. 7 shows how this zone evolves with time for different critical effective strains $J_{0}$. As time increases and the material creeps, the cavity expands and overall deformation becomes larger, so the damage zone also expands. Also, the smaller the critical effective strain, the faster the physical bonds breaks under the same stress, and the softer the material, which leads to larger damage zone sizes. Thus, the damage zone size scales inversely with $J_{0}$.

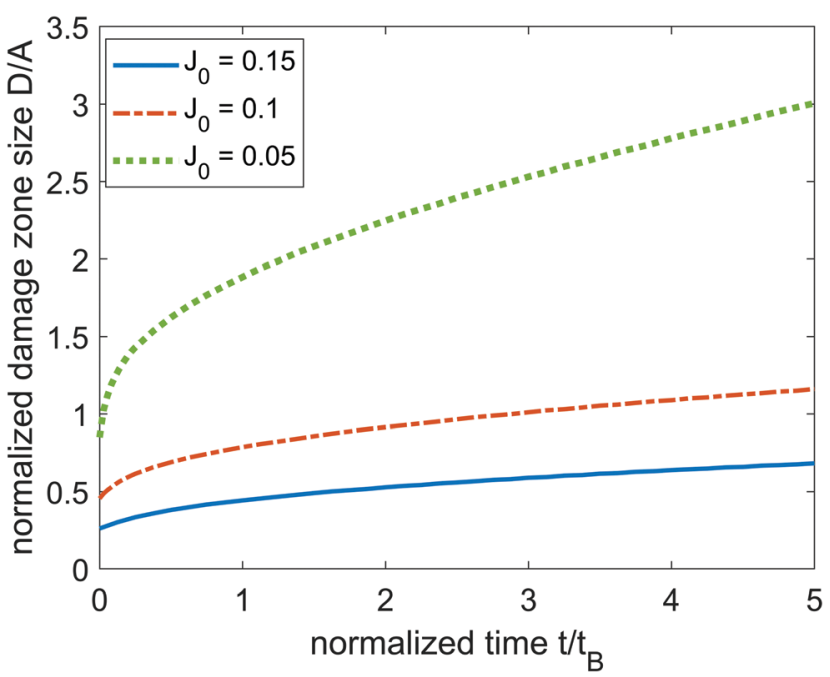

Fig. 7 Time evolution of the normalized damage zone size ( $A$ is the initial cavity radius). The damage zone expands as time increases and the material creeps. Smaller $J_{0}$ leads to larger damage zones.

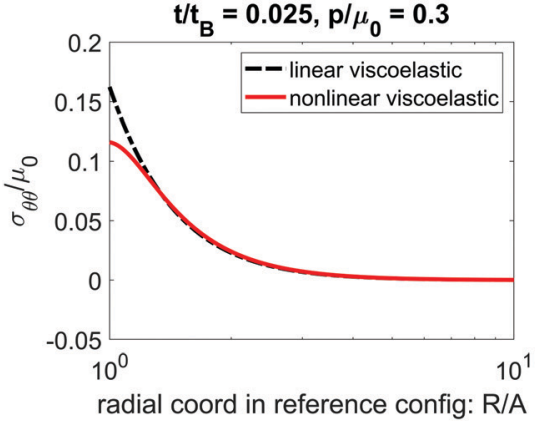

(a)

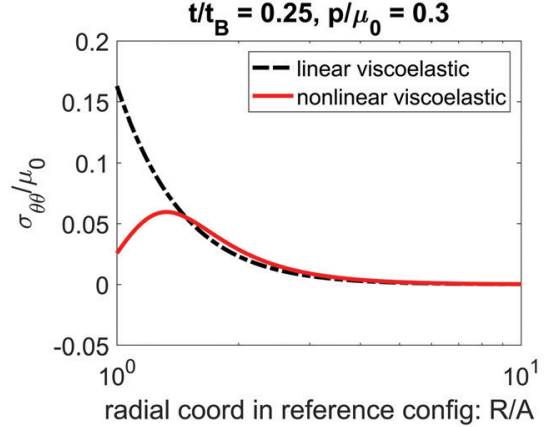

(b)

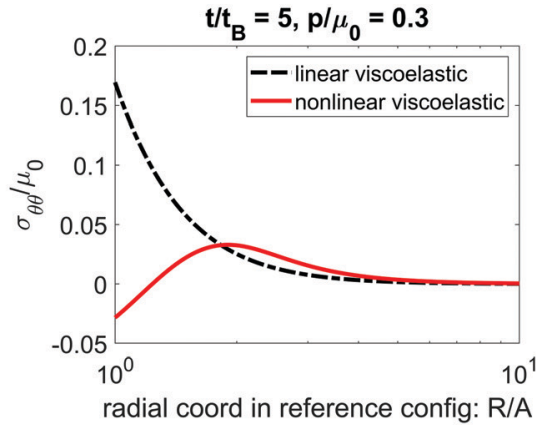

(c)

Fig. 6 Distribution of normalized hoop stress $\sigma_{\theta \theta} / \mu_{0}$ at different normalized times $t / t_{\mathrm{B}}=0.025,0.25,5$ of a linear and nonlinear viscoelastic solid. Material parameters are given in eqn (14), and the constant internal pressure is $p / \mu_{0}=0.3$ for all $t>0$. 
4.3 Zero degree peel test in a linear viscoelastic/nonlinear viscoelastic solid

The cavity problem shows that significant stress concentration and shielding can occur in structures without cracks. In this section we study crack shielding by considering a simple one dimensional crack model, the zero degree peel test. This test is often used by the adhesive industry to study the resistance of pressure sensitive tapes to shear. ${ }^{49}$ The geometry is shown in Fig. 8. A thin adhesive layer of thickness $h$ is sandwiched between a rigid substrate and a thin stiff backing layer. The backing layer is linear elastic with plane strain modulus $E_{\mathrm{B}}$ and has thickness $h_{\mathrm{B}}$. The layers are infinite in the $x$ and $z$ direction and we assume plane strain deformation so fields are independent of $z$, the out of plane coordinate. The peel arm has length $L$ and occupies $-L<x<0$. The adhesive layer is viscoelastic (linear or nonlinear) and is perfectly bonded to the stiff backing layer along the interface $y=h,-L<x<\infty$. The adhesive is bonded to the rigid substrate along the interface at $y=0$ and $x>0$. However, along $y=0,-L<x<0$ it is traction free. This traction free surface can be viewed as the face of an interface crack between the adhesive and the rigid substrate (see Fig. 8). The crack tip is at $x=y=0$. The tape is pulled horizontally in the negative $x$ direction in a zero degree peel test.

To simplify the calculations, we determine the stress and strain in the adhesive layer using a one dimensional shear-lag model. Details of this model can be found in Hui et al. ${ }^{50}$ Here we briefly summarize ideas that are relevant to this work. In this model, the linear elastic backing layer can only support in-plane tension, the spatial gradient of this tension is balanced by the interfacial shear stress $\tau$ exerted by the adhesive layer, which is related to the shear strain $\gamma$ of the adhesive layer by a viscoelastic model. The shear strain $\gamma$ is related to the horizontal displacement of the adhesive/backing interface $u$ by $\gamma=u / h$. A limitation of this model is that the singular field at the crack tip is regularized by the shear-lag approximation. Nevertheless, there is still a stress concentration at the "crack" tip and we can study how nonlinear viscoelasticity alters this stress concentration.

We study crack shielding near the crack tip at $x=y=0$. In the following, we normalized all stress quantity by the small strain shear modulus of the networks, $\mu_{\text {net }}$. All distances are normalized by a length parameter $l_{\mathrm{lt}} \equiv \sqrt{E_{\mathrm{B}}{ }^{*} h_{\mathrm{B}} h / \mu_{\text {net }}}$; it represents the length of adhesive that carries shear and is called the effective load transfer length. In practice, $E_{\mathrm{B}}{ }^{*} / \mu_{\text {net }} \approx 10^{4}-10^{5}$, so this length is on the order of hundreds of tape thickness. Normalized quantities will be topped with a bar.

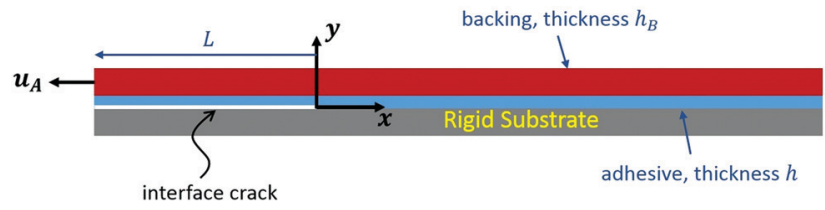

Fig. 8 Schematic of the zero degree peel test. $L$ is the length of the peel $\operatorname{arm}, L \gg h, h_{\mathrm{B}}$. The air gap between the peel arm and the substrate can be viewed as an interface crack.
As shown in Hui, ${ }^{50}$ the governing equation for the shear train in the adhesive layer $\gamma$ is

$$
\frac{\partial^{2} \gamma}{\partial \bar{x}^{2}}=\bar{\tau}(\bar{x}, t) \quad \bar{x}>0
$$

where $\bar{\tau}=\tau / \mu_{\text {net }}$ is the normalized shear stress in the adhesive layer and $\bar{x}=x / l_{\mathrm{lt}}$ is the normalized $x$ coordinate. If the adhesive is linear viscoelastic, then the shear stress is related to the shear strain by

$$
\bar{\tau}(\bar{x}, t)=\frac{1}{\mu_{\text {net }}} \int_{0}^{t} \mu(t-s) \frac{\partial \gamma(\bar{x}, s)}{\partial s} \mathrm{~d} s
$$

where $\mu$ is given by eqn (10a). In the nonlinear theory, we specialize eqn (13) for simple shear deformation and find:

$$
\bar{\tau}(\bar{x}, t)=\rho \gamma(\bar{x}, t)+\int_{-\infty}^{t} \chi(\bar{x}, s) \phi_{\mathrm{B}}(s, t)[\gamma(\bar{x}, t)-\gamma(\bar{x}, s)] \mathrm{d} s .
$$

The adhesive layer is sheared by imposing a constant displacement rate $\dot{u}_{\mathrm{A}}$ on the stiff backing layer at $x=-L$ for $t \geq 0$. We assumed that loading commences at $t=0$ so that $\gamma(\bar{x}, t \leq 0)=0$. Using force balance, the displacement boundary condition

$$
\gamma\left(\bar{x}=-L / l_{\mathrm{lt}}, t>0\right)=\dot{u}_{\mathrm{A}} t / h
$$

can be written as:

$$
\begin{gathered}
E_{\mathrm{B}}{ }^{*} h_{\mathrm{B}}\left[\frac{\dot{u}_{\mathrm{A}} t-u(x=0, t)}{L}\right]=\int_{0}^{\infty} \tau(x) \mathrm{d} x \\
\Rightarrow \frac{l_{\mathrm{lt}}}{L}\left[\gamma_{\mathrm{A}}-\gamma(\bar{x}=0, t)\right]=\int_{0}^{\infty} \bar{\tau}(\bar{x}) \mathrm{d} \bar{x}
\end{gathered}
$$

where $\gamma_{\mathrm{A}}=\dot{u}_{\mathrm{A}} t / h$.

The solution of the linear theory is obtained by numerically solving eqn (21) with the shear stress on the RHS given by eqn (22). The initial condition is:

$$
\gamma(\bar{x}, \bar{t}=0)=0
$$

The boundary conditions are eqn (24a) and the condition that strain vanishes at infinity. The solution of the nonlinear theory is obtained by numerically solving eqn (21) and (24b) with the RHS of eqn (21) given by eqn (23). The parameters used in all the simulations are given by eqn (14). Fig. 9a-d plots the evolution of the shear stress and strain at different times for the linear and nonlinear case. Recall that the linear and nonlinear model have the same small strain behavior as well as identical long and short time modulus. For the nonlinear solid, it is clear that there is a region near the crack tip where the shear stress is lower than the linear solid. The size of this "damage" zone increases with time. Time evolution of the maximum shear stress at the crack tip is shown in Fig. 10a. More importantly, the nonlinear viscoelastic solid has a much smaller maximum stress as the strain or time increase. Reduction in stress confirms the shielding effect due to the rapid breaking of physical cross-links. The stress reduction factor $\left(\bar{\tau}_{\text {max }}\right)_{\text {nonlinear }} /\left(\bar{\tau}_{\text {max }}\right)_{\text {linear }}$ is shown in Fig. 10b.

One way of rationalizing the results in Fig. $9 \mathrm{a}-\mathrm{d}$ is to use the concept of "yield stress" introduced earlier. The "yield stress" $\sigma_{\mathrm{Y}}$ in this simulation is $\mu_{\text {net }} J_{0}=0.1 \mu_{\text {net. }}$ In Fig. 9a and b, the maximum shear stress is less than $\sigma_{\mathrm{Y}}$, for this case there is 

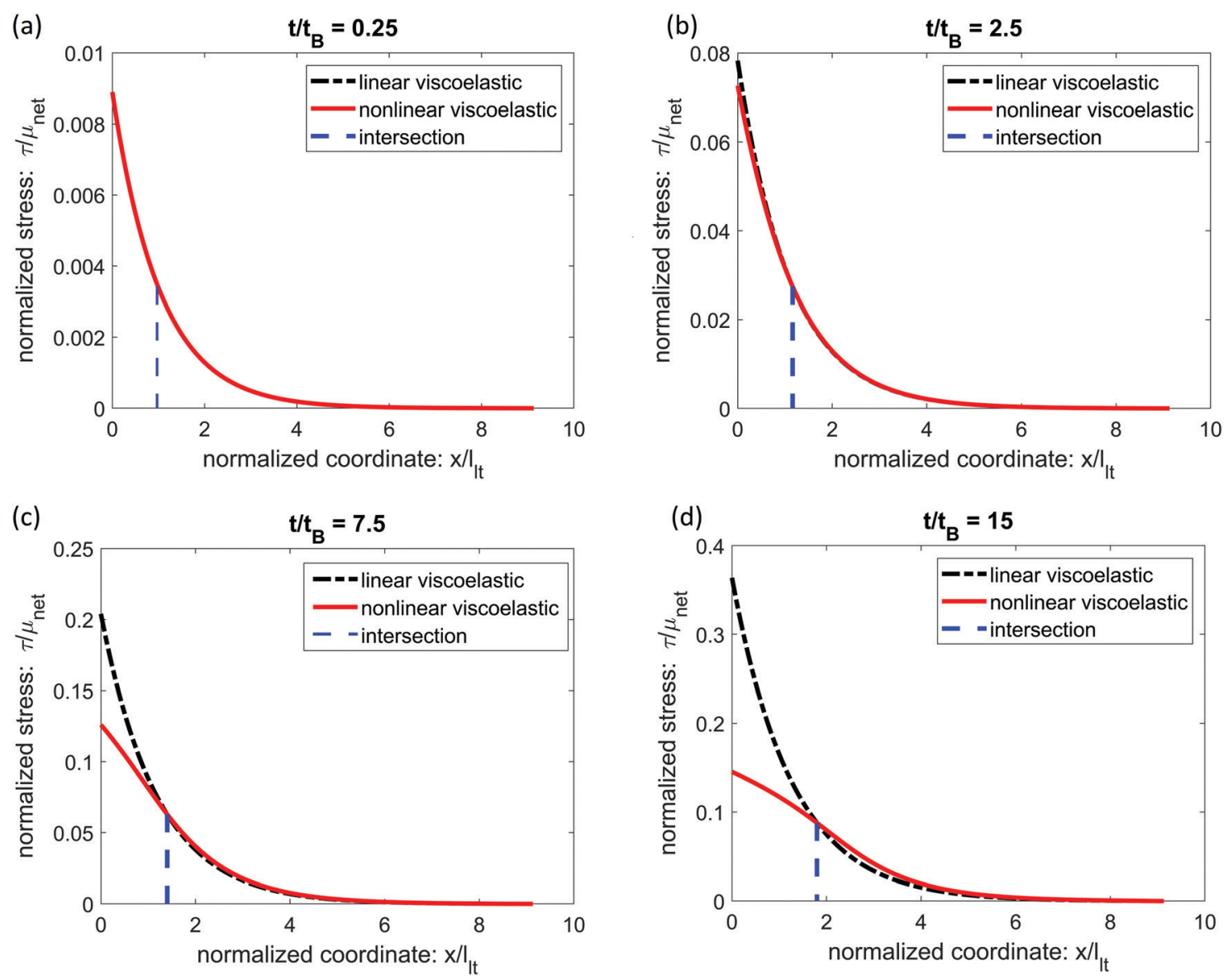

Fig. 9 Shear stress profile in the zero-degree peel test at different times: $t / t_{B}=0.25,2.5,7.5,15$. The applied displacement rate divided by the tape thickness is $\dot{u}_{A} / h=1 / 25 t_{B}$. The black dash-dotted curves are the stress profiles for the linear case, while the red solid curves are for the nonlinear case. The blue dashed line indicates the region where the stress of the nonlinear solid is lower than the linear one.

practically no difference between the linear and nonlinear model. However, when the maximum shear stress increases beyond $0.1 \mu_{\text {net }}$, the damage zone appears and the cross-over point between linear and nonlinear theory occurs slightly below $0.1 \mu_{\text {net }}$.

\section{Summary and discussion}

Using a physical model based on bond breaking and healing kinetics, we present arguments and examples to highlight some of the pitfalls of employing LVF theory to model fracture.

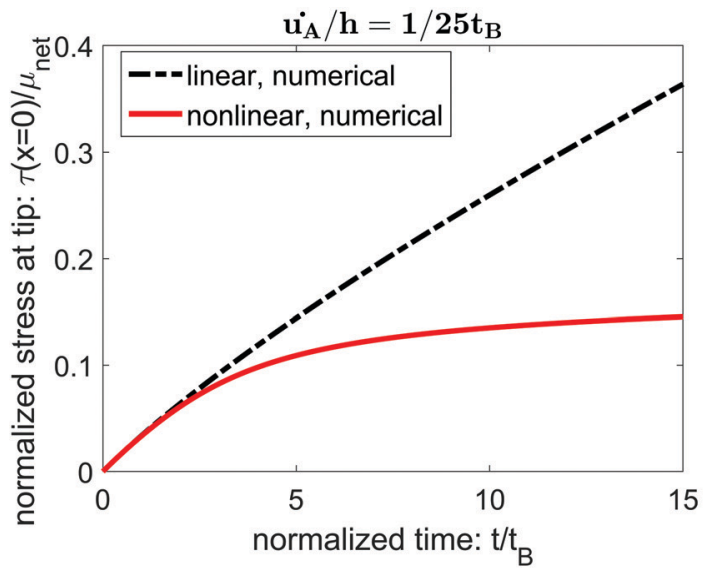

(a)

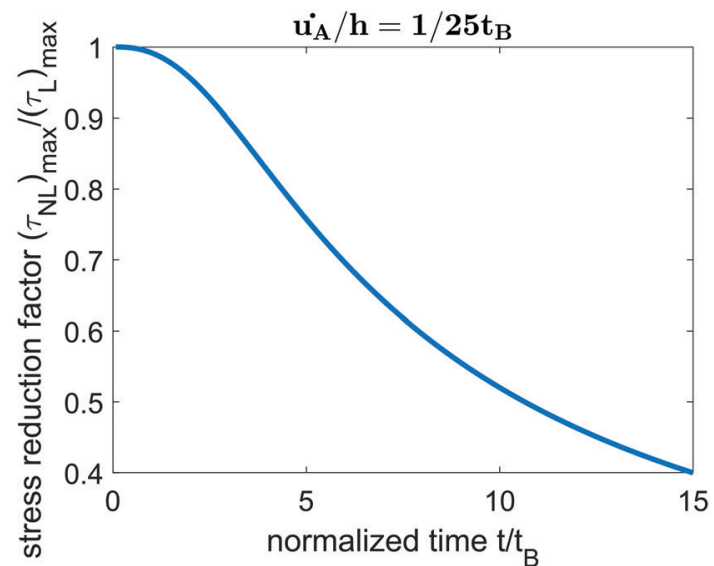

(b)

Fig. 10 (a) Maximum shear stress at $\bar{x}=0$; (b) stress reduction factor $\left(\bar{\tau}_{\text {max }}\right)_{\text {nonlinear }} /\left(\bar{\tau}_{\text {max }}\right)_{\text {linear }}$ as a function of normalized time. 
We suggest that many of the paradoxes and difficulties associated with LVF can be resolved by allowing the relaxation times to be dependent on stress/strain histories. For example, materials under high strains (near the crack tip) relax much faster, and the stresses carried by these damaged materials are substantially lower than a material that is linear viscoelastic, and this results in stress shielding. We illustrate this crack-shielding effect by surrounding the crack tip by a soft elastic core. To analyze this crack-shielding effect more quantitatively, we extend a linear viscoelastic model to a nonlinear one by including stress-dependent bond breaking kinetics; both models have the same short and long time behavior, and are identical under small strains. We use a cavity subjected to internal pressure and the zero degree peel test to highlight the relevant physics.

At first glance, it seems counter-intuitive that the breakdown of most the physical bonds near the crack tip would not immediately lead to failure. This is because fracture requires the failure of the entire network, which means that chemical crosslinked network will have to fail too. However, as long as damage is confined, the chemical crosslinked network will not failure due to shielding. Of course, eventually the damage zone will be large enough and the chemical network will fail, because the polymer chains reach a maximum extensibility that leads to fracture. This is analogous to the situation of the tough rate independent double network gel discovered by Gong. ${ }^{51}$ As noted by Brown, ${ }^{52}$ the increase in toughness is due to the breaking of the stiff network and is directly proportional to the size of the damage zone (which is controlled also by the maximum extensibility of the soft network).

The absence of a length scale for the dissipation zone near the crack tip is a severe limitation of the linear theory. In LVF theory, a length scale is introduced using a cohesive zone model $^{5,6}$ to eliminate the stress singularity. The size of this zone scales with the size of the dissipative zone, which is found to be of atomic dimensions. In the nonlinear theory, the rapid breaking of bonds near the crack tip introduces a physically relevant length scale in the bulk that can be identified with dissipation. Specifically, the nonlinear viscoelastic model used in this work exhibits a rate dependent "yield stress". Many tough gels as well as pressure sensitive adhesives exhibit such "yield" behavior, see for example ref. 23, 53 and 54. In our model, the physical cross-links break much faster when they are stretched to a critical strain, thus the material "yields" at this critical strain, but the stress associated with this "yield" depends on the loading history (such as loading rate). Although the actual yield stress in shear $\sigma_{\mathrm{Y}}$ is history dependent, an upper estimate of $\sigma_{\mathrm{Y}}$ is roughly $\mu_{\text {net }} J_{0}$, where $\mu_{\text {net }}$ is the modulus of the undamaged network and $J_{0}$ is the effective critical strain where chain breaking rate increases rapidly. Similar to elasticplastic fracture in metals where a plastic zone size can be defined, this time dependent yield stress introduces naturally a new length scale in the fracture problem. For example, let's assume small scale yielding, that is, that damage is confined to a region that is very small in comparison with crack length, so that the stress intensity factor $K_{\mathrm{I}}$ of the linear theory controls the local nonlinear fields. Then the size of the damage zone can be estimated by setting the effective strain equals to $J_{0}$. Roughly,

$$
\frac{K_{\mathrm{I}}}{\mu(t) \sqrt{2 \pi D}} \approx J_{0} \Rightarrow D \approx \frac{K_{\mathrm{I}}^{2}}{2 \pi\left[\mu(t) J_{0}\right]^{2}}
$$

where $\mu(t)$ is the relaxation modulus. The smallest $D$ is obtained by setting $\mu(t)=\mu_{0}$ in eqn (26). Let us compare $D$ with the cohesive zone size $d \approx K_{\mathrm{I}}^{2} / \sigma_{0}{ }^{2}$ in LVF. A rough estimate is that the cohesive stress $\sigma_{0}$ equals the average stress needed to break all the chains crossing a unit surface area. In the appendix, we show that this is roughly $4 \mathrm{GPa}$. This is much greater than $\sigma_{\mathrm{Y}} \approx \mu_{\text {net }} J_{0}$. For example, the undamaged network modulus of the polyampholyte gel ${ }^{23}$ is roughly $1 \mathrm{MPa}$, and $J_{0} \approx 0.1$, thus $\sigma_{\mathrm{Y}} \approx 0.1 \mathrm{MPa}$. For the same stress intensity factor $K_{\mathrm{I}}$, the ratio $D / d \approx\left(\sigma_{0} / \sigma_{\mathrm{Y}}\right)^{2} \approx 10^{8}$. Thus the dissipation zone size is at least on the order of mms which is appropriate for soft, tough materials. Indeed, in the cavity example studied the damage zone sizes are comparable to the characteristic size of the geometry.

We emphasize that nonlinear viscoelastic behavior can arise from a broad spectrum of mechanisms other than the stress dependent bond breaking and healing kinetics we considered in this work. For example, Elziere et al. ${ }^{55}$ have shown that the nonlinear viscoelastic behavior of poly(vinylbutyral), a tough polymer used for the interlayer of laminated glass, is controlled by a completely different mechanism. In this polymer, viscoelastic flow is inhibited by high strains. The dissipative zone size in this case can be identified with the hardening region near the crack tip. A series of nanostructured waterborne adhesives designed with this nonlinear softening mechanism are intrinsically shielded from crack propagation. ${ }^{54}$ Also, there are alternative bond breaking models where the bond breaking rate is affected by the stretch rate rather than the stretch experience by the polymer strands. ${ }^{56,57}$

The fact that the cohesive zone size does not scale with the dissipative zone size does not mean that the cohesive zone model is not useful. It merely points to the fact that dissipation and failure processes involve different physics. These different mechanisms do not mean that these processes are weakly coupled. Indeed, it is commonly assumed that the cohesive properties are independent of continuum crack tip fields while in reality there are significant interactions between separation process and local viscoelastic deformation. The coupling of these two processes is a challenging problem that requires further study.

Finally, we address an excellent question raised by an anonymous reviewer: what happens to the concept of fracture energy which is so widely used in fracture mechanics? Although experimentalists use fracture mechanics concepts as an approximation in weakly viscoelastic materials, the use of fracture energy to quantify rate dependent fracture in general is full of theoretical difficulties. This is because energy release rate is not well defined in viscoelastic solids (linear or nonlinear) except under very special circumstances, e.g. steady state crack growth under condition where the material away from the crack tip is fully relaxed and therefore elastic. A difficulty is that dissipation occurs everywhere in the specimen, independent of whether a 
material point is close to or far away from the crack tip. In addition, the mechanical energy at a material point depends on the entire history of loading and it is difficult to separate this energy into a dissipative and a non-dissipative part. Even if this can be done, the amount of energy dissipated can be specimen dependent and strongly coupled to the intrinsic energy of fracture. Due to these difficulties, we have avoided the use of fracture energy and focus on the effect of nonlinear viscoelasticity on dissipation near a crack tip.

\section{Conflicts of interest}

There are no conflicts to declare.

\section{Appendix}

\section{A1. Healing rate in the nonlinear viscoelastic model and strain energy density}

As was described in our previous work, ${ }^{44}$ the healing rate $\chi(t)$ is assumed to be directly proportional to $n_{\mathrm{b}}(t)$ which is the number of physical cross-links per unit reference volume that are broken at the current time, i.e.,

$$
\chi(t)=\frac{1}{t_{\mathrm{H}}} \frac{n_{\mathrm{b}}(t)}{N_{0}},
$$

where $t_{\mathrm{H}}$ is a characteristic time for healing which is assumed to be insensitive to chain stretch, and $N_{0}$ is the total number of bonds. Because breaking rate is dependent on strain history, the steady state assumption of the linear theory where breaking and healing rates are equal is no longer valid. The number of unconnected bonds $n_{\mathrm{b}}$ in eqn (A1) is dependent on the strain history:

$$
N_{0}=N_{1}+N_{2}(t)+n_{\mathrm{b}}(t)
$$

where $N_{1}=\rho N_{0}$ is the number of chemically cross-linked bonds and $N_{2}(t)$ is the number of surviving physical bonds. We evaluate $N_{2}(t)$ by summing all the healed bonds that survive to current time $t$. This is calculated by noting that the number of physical bonds that are borne between $t^{\prime}$ and $t^{\prime}+\mathrm{d} t^{\prime}$ is by definition $\chi\left(t^{\prime}\right) N_{0} \mathrm{~d} t^{\prime}$, so the number of bonds that are connected is the integral of $\chi\left(t^{\prime}\right) N_{0} \mathrm{~d} t^{\prime} \cdot \phi_{\mathrm{B}}\left(t^{\prime}, t\right)$ from $t^{\prime}=-\infty$ to the current time, i.e.,

$$
N_{2}(t)=N_{0} \int_{-\infty}^{t} \chi\left(t^{\prime}\right) \phi_{\mathrm{B}}\left(t^{\prime}, t\right) \mathrm{d} t^{\prime} .
$$

Combining eqn (A1), (A2) and (A3a) and divide the equation by $N_{0}$, we have

$$
1-\rho=\int_{-\infty}^{t} \chi\left(t^{\prime}\right) \phi_{\mathrm{B}}\left(t^{\prime}, t\right) \mathrm{d} t^{\prime}+\chi\left(t^{\prime}\right) t_{\mathrm{H}}
$$

The total strain energy density at a material point in the stress-free reference configuration is

$$
W(t)=\rho W_{0}\left[H\left(t^{\prime}=0, t\right)\right]+\int_{-\infty}^{t} \chi\left(t^{\prime}\right) \phi_{\mathrm{B}}\left(t^{\prime}, t\right) W_{0}\left[H\left(t^{\prime}, t\right)\right] \mathrm{d} t^{\prime}
$$

The 1 st term is the strain energy per unit reference volume of the chemical cross-linked network. The 2nd term is the strain energy per unit reference volume carried by the physical bonds. To see this, we note that $\chi\left(t^{\prime}\right) \phi_{\mathrm{B}}\left(t^{\prime}, t\right) \mathrm{d} t^{\prime}$ is the molar fraction of physical chains that reattaches between $\left(t^{\prime}, t^{\prime}+\mathrm{d} t^{\prime}\right)$ and survives till $t$. Since the strain energy density carried by these chains is $W_{0}\left[H\left(t^{\prime}, t\right)\right]$, the total strain energy density carried by all physical chains is the integral of $\chi\left(t^{\prime}\right) \phi_{\mathrm{B}}\left(t^{\prime}, t\right) \mathrm{d} t^{\prime} \cdot W_{0}\left[H\left(t^{\prime}, t\right)\right]$ from $t^{\prime}=-\infty$ to the current time $t$. The relation between Cauchy (true) stress $\boldsymbol{\sigma}$ and history of deformation can be computed using eqn (A4) and following the Coleman-Noll procedure.

A2. Cavity growth in a linear viscoelastic solid: eqn (19) and (20)

Using $\mathbf{P}=\boldsymbol{\sigma} \mathbf{F}^{-T}$, eqn (10a) and (11), and the deformation gradient is the identity tensor for time less than zero, the first Piola-Kirchoff stress of the linear viscoelastic solid is

$$
\begin{aligned}
\mathbf{P}= & -q\left(\mathbf{F}^{0 \rightarrow t}\right)^{-T}+\mu(t) \mathbf{F}^{0 \rightarrow t} \\
& +\int_{0}^{t} \frac{\partial \mu\left(t-t^{\prime}\right)}{\partial t^{\prime}} \mathbf{F}^{t^{\prime} \rightarrow t}\left(\mathbf{F}^{0 \rightarrow t^{\prime}}\right)^{-T} \mathrm{~d} t^{\prime}
\end{aligned}
$$

where $\mu(t)$ is defined by eqn (10a). The deformation gradient $\mathbf{F}^{t^{\prime} \rightarrow t}=\mathbf{F}^{0 \rightarrow t}\left[\mathbf{F}^{0 \rightarrow t^{\prime}}\right]^{-1}$ is

$$
\begin{aligned}
\mathbf{F}^{t^{\prime} \rightarrow t} & =\left[\begin{array}{ccc}
\frac{r_{, R}(R, t)}{r_{, R}\left(R, t^{\prime}\right)} & 0 & 0 \\
0 & \frac{r(R, t)}{r\left(R, t^{\prime}\right)} & 0 \\
0 & 0 & \frac{r(R, t)}{r\left(R, t^{\prime}\right)}
\end{array}\right] \\
= & {\left[\begin{array}{ccc}
\frac{r^{2}\left(R, t^{\prime}\right)}{r^{2}(R, t)} & 0 & 0 \\
0 & \frac{r(R, t)}{r\left(R, t^{\prime}\right)} & 0 \\
0 & 0 & \frac{r(R, t)}{r\left(R, t^{\prime}\right)}
\end{array}\right] }
\end{aligned}
$$

where we have used the incompressibility condition. The $r_{, \mathrm{R}}$ denotes partial derivative with respect to $R$.

$$
\begin{aligned}
& \operatorname{det} \mathbf{F}=\frac{\mathrm{d} r}{\mathrm{~d} R} \cdot \frac{r}{R} \cdot \frac{r}{R}=1 \Rightarrow r^{2} \mathrm{~d} r=R^{2} \mathrm{~d} R \Rightarrow r(R, t) \\
& =\sqrt[3]{R^{3}+a^{3}(t)-A^{3}}=\sqrt[3]{R^{3}+c(t)}
\end{aligned}
$$

where $a(t)$ is the radius of the cavity at time $t$ and

$$
c(t) \equiv a^{3}(t)-A^{3}=r^{3}(R, t)-R^{3} \Rightarrow r^{3}(R, t)=R^{3}+c(t) .
$$

Because of symmetry, the only non-trivial equilibrium equations is in the radial direction. This equation, in the reference configuration is

$$
\frac{\partial P_{R R}}{\partial R}+\frac{2 P_{R R}-2 P_{\Theta \Theta}}{R}=0 .
$$


Substituting eqn (A5), (A6) into (A7) and using (A6c), the first term is

$$
\begin{aligned}
\frac{\partial P_{R R}}{\partial R}= & -\frac{\partial q}{\partial R} \frac{r^{2}(R, t)}{R^{2}}-2 q\left[-\frac{r^{2}(R, t)}{R^{3}}+\frac{1}{r(R, t)}\right] \\
& +2 \mu(t)\left[\frac{R}{r^{2}(R, t)}-\frac{R^{4}}{r^{5}(R, t)}\right] \\
& +\int_{0}^{t} \frac{\partial \mu\left(t-t^{\prime}\right)}{\partial t^{\prime}}\left[\frac{4 r\left(R, t^{\prime}\right)}{r^{2}(R, t)}-\frac{2 r^{4}\left(R, t^{\prime}\right)}{r^{5}(R, t)}-\frac{2 r^{4}\left(R, t^{\prime}\right)}{r^{2}(R, t) R^{3}}\right] \mathrm{d} t^{\prime}
\end{aligned}
$$

The second term in eqn (A7) is

$$
\begin{aligned}
\frac{2 P_{R R}-2 P_{\Theta \Theta}}{R}= & 2 q\left[\frac{1}{r(R, t)}-\frac{r^{2}(R, t)}{R^{3}}\right] \\
& +2 \mu(t)\left[\frac{R}{r^{2}(R, t)}-\frac{r(R, t)}{R^{2}}\right] \\
& +2 \int_{0}^{t} \frac{\partial \mu\left(t-t^{\prime}\right)}{\partial t^{\prime}}\left[\frac{r^{4}\left(R, t^{\prime}\right)}{r^{2}(R, t) R^{3}}-\frac{r(R, t)}{r^{2}\left(R, t^{\prime}\right)}\right] \mathrm{d} t^{\prime}
\end{aligned}
$$

Adding (A8a), (A8b) and (A7) simplifies to

$$
\begin{aligned}
& -\frac{\partial q}{\partial R} \frac{r^{2}(R, t)}{R^{2}}+\mu(t)\left[\frac{4 R}{r^{2}(R, t)}-\frac{2 R^{4}}{r^{5}(R, t)}-\frac{2 r(R, t)}{R^{2}}\right] \\
& +\int_{0}^{t} \frac{\partial \mu\left(t-t^{\prime}\right)}{\partial t^{\prime}}\left[\frac{4 r\left(R, t^{\prime}\right)}{r^{2}(R, t)}-\frac{2 r^{4}\left(R, t^{\prime}\right)}{r^{5}(R, t)}-\frac{2 r(R, t)}{r^{2}\left(R, t^{\prime}\right)}\right] \mathrm{d} t^{\prime}=0 .
\end{aligned}
$$

It can be shown (e.g. by direct differentiation) that the solution of (A9a) is

$$
\begin{aligned}
q= & -\mu(t)\left[\frac{3}{2} \frac{R}{r(R, t)}+\frac{c(t)}{2} \frac{R}{r^{4}(R, t)}\right] \\
& -\int_{0}^{t} \frac{\partial \mu\left(t-t^{\prime}\right)}{\partial t^{\prime}}\left[\frac{3}{2} \frac{r\left(R, t^{\prime}\right)}{r(R, t)}+\frac{c(t)-c\left(t^{\prime}\right)}{2} \frac{r\left(R, t^{\prime}\right)}{r^{4}(R, t)}\right] \mathrm{d} t^{\prime} \\
& +q_{0}(t)
\end{aligned}
$$

where $c(t)$ is defined by (A6c) and $q_{0}(t)$ is an arbitrary function of time only. The PK-I radial stress is obtained by substituting (A9b) into (A5)

$$
\begin{aligned}
\mathbf{P}_{R R}(R, t)= & \mu(t)\left[\frac{2 r(R, t)}{R}+\frac{R^{2}}{2 r^{2}(R, t)}\right] \\
& +\int_{0}^{t} \frac{\partial \mu\left(t-t^{\prime}\right)}{\partial t^{\prime}}\left[\frac{2 r\left(R, t^{\prime}\right) r(R, t)}{R^{2}}+\frac{r^{4}\left(R, t^{\prime}\right)}{2 r^{2}(R, t) R^{2}}\right] \mathrm{d} t^{\prime} \\
& -q_{0}(t) \frac{r^{2}(R, t)}{R^{2}}
\end{aligned}
$$

We determine $q_{0}(t)$ from (A9c) using the far field boundary condition

$$
\begin{aligned}
& \left.\mathbf{P}_{R R}(t)\right|_{R \rightarrow \infty}=0,\left.\quad \frac{r(R, t)}{R}\right|_{R \rightarrow \infty} \\
& =1 \Rightarrow \frac{5}{2} \mu(t)+\frac{5}{2} \int_{0}^{t} \frac{\partial \mu\left(t-t^{\prime}\right)}{\partial t^{\prime}} \mathrm{d} t^{\prime}-q_{0}(t)=0 \\
& \Rightarrow q_{0}(t)=\frac{5}{2}\left[\mu(t)+\int_{0}^{t} \frac{\partial \mu\left(t-t^{\prime}\right)}{\partial t^{\prime}} \mathrm{d} t^{\prime}\right]=\frac{5}{2} \mu_{0}
\end{aligned}
$$

Combining eqn (A9b) and (A10) along with the deformation gradient given by eqn (A6a), the true stresses are

$$
\begin{aligned}
& \sigma_{r r}(r, t)=\mu(t)\left[\frac{2 R}{r(R, t)}+\frac{R^{4}}{2 r^{4}(R, t)}\right] \\
&-\frac{5}{2} \mu_{0}+\int_{0}^{t} \frac{\partial \mu\left(t-t^{\prime}\right)}{\partial t^{\prime}}\left[\frac{2 r\left(R, t^{\prime}\right)}{r(R, t)}+\frac{r^{4}\left(R, t^{\prime}\right)}{2 r^{4}(R, t)}\right] \mathrm{d} t^{\prime} \\
& \sigma_{\theta \theta}(r, t)=\sigma_{\phi \phi}(r, t) \\
&=\mu(t)\left[\frac{2 R}{r(R, t)}-\frac{R^{4}}{2 r^{4}(R, t)}+\frac{r^{2}(R, t)}{R^{2}}\right]-\frac{5}{2} \mu_{0} \\
&+\int_{0}^{t} \frac{\partial \mu\left(t-t^{\prime}\right)}{\partial t^{\prime}}\left[\frac{2 r\left(R, t^{\prime}\right)}{r(R, t)}-\frac{r^{4}\left(R, t^{\prime}\right)}{2 r^{4}(R, t)}+\frac{r^{2}(R, t)}{r^{2}\left(R, t^{\prime}\right)}\right] \mathrm{d} t^{\prime}
\end{aligned}
$$

The inner radius $a(t)$ is determined by the boundary condition

$$
\begin{aligned}
& \left.\sigma_{r r}\right|_{r=a}=-p \\
& \Rightarrow \mu(t)\left[\frac{2 A}{a(t)}+\frac{A^{4}}{2 a^{4}(t)}\right]-\frac{5}{2} \mu_{0} \\
& +\int_{0}^{t} \frac{\partial \mu\left(t-t^{\prime}\right)}{\partial t^{\prime}}\left[\frac{2 a\left(t^{\prime}\right)}{a(t)}+\frac{a^{4}\left(t^{\prime}\right)}{2 a^{4}(t)}\right] \mathrm{d} t^{\prime}=-p
\end{aligned}
$$

We normalize all lengths by $A$, e.g. $\bar{r} \equiv r / A$. Then eqn (A12a) reduces to

$$
\begin{aligned}
& \mu(t)\left[\frac{2}{\bar{a}(t)}+\frac{1}{2 \bar{a}^{4}(t)}\right] \\
& +\int_{0}^{t} \frac{\partial \mu\left(t-t^{\prime}\right)}{\partial t^{\prime}}\left[\frac{2 \bar{a}\left(t^{\prime}\right)}{\bar{a}(t)}+\frac{\bar{a}^{4}\left(t^{\prime}\right)}{2 \bar{a}^{4}(t)}\right] \mathrm{d} t^{\prime}=\frac{5}{2} \mu_{0}-p
\end{aligned}
$$

which is eqn (19) divided by 2 . The instantaneous response due to the sudden pressure load is determined by setting $t=0$ in eqn (A12b):

$$
\mu_{0}\left[\frac{2}{\bar{a}(0)}+\frac{1}{2 \bar{a}^{4}(0)}\right]=\frac{5}{2} \mu_{0}-p \Rightarrow \frac{1}{\bar{a}^{4}(0)}+\frac{4}{\bar{a}(0)}=5-\frac{2 p}{\mu_{0}} .
$$

Eqn (A13) is the same as the elastic solution given by eqn (17b) of a neo-Hookean solid with small strain modulus $\mu=\mu_{0}$.

\section{A3. Nonlinear case}

In the nonlinear case, the survivability function becomes

$$
\phi_{\mathrm{B}}\left(t^{\prime}, t\right)=\left\{1+\frac{\alpha_{\mathrm{B}}-1}{t_{\mathrm{B}}} \int_{t^{\prime}}^{t}\left[1+\frac{H\left(t^{\prime}, s\right)-3}{J_{0}^{2}}\right]^{m} \mathrm{~d} s\right\}^{\frac{1}{1-\alpha_{\mathrm{B}}}}
$$




$$
H\left(t^{\prime}, s\right)=\frac{r^{4}\left(R, t^{\prime}\right)}{r^{4}(R, s)}+2 \frac{r^{2}(R, s)}{r^{2}\left(R, t^{\prime}\right)} .
$$

Note that $\phi_{\mathrm{B}}\left(t^{\prime}, t\right)$ is a function of radial coordinate $R$, and so is the healing rate $\chi$ which is determined by eqn (A3b). To explicitly express this dependence, we denote these quantities as $\phi_{\mathrm{B}}\left(R, t^{\prime}, t\right), \chi(R, t)$, and $H\left(R, t^{\prime}, s\right)$ respectively. From eqn (13) and using $\mathbf{P}=\boldsymbol{\sigma} \mathbf{F}^{-T}$, and assuming that there is no load for $t<0$, the nonzero PK-I stress components for the nonlinear viscoelastic solid are

$$
\begin{aligned}
P_{R R}= & -q \frac{r^{2}(R, t)}{R^{2}}+\mu\left[\rho+\chi_{\infty} \frac{t_{\mathrm{B}}}{2-\alpha_{\mathrm{B}}} \phi_{\mathrm{B}}(R, 0, t)^{\left(2-\alpha_{\mathrm{B}}\right)}\right] \frac{R^{2}}{r^{2}(R, t)} \\
& +\mu \int_{0}^{t} \chi\left(R, t^{\prime}\right) \phi_{\mathrm{B}}\left(R, t^{\prime}, t\right) \frac{r^{4}\left(R, t^{\prime}\right)}{r^{2}(R, t) R^{2}} \mathrm{~d} t^{\prime} \\
P_{\Theta \Theta}= & P_{\Phi \Phi} \\
= & -q \frac{R}{r(R, t)}+\mu\left[\rho+\chi_{\infty} \frac{t_{\mathrm{B}}}{2-\alpha_{\mathrm{B}}} \phi_{\mathrm{B}}(R, 0, t)^{\left(2-\alpha_{\mathrm{B}}\right)}\right] \frac{r(R, t)}{R} \\
& +\mu \int_{0}^{t} \chi\left(R, t^{\prime}\right) \phi_{\mathrm{B}}\left(R, t^{\prime}, t\right) \frac{r(R, t) R}{r^{2}\left(R, t^{\prime}\right)} \mathrm{d} t^{\prime}
\end{aligned}
$$

and the equilibrium eqn (A7) becomes

$$
\begin{aligned}
0= & -\frac{\partial q}{\partial R} \frac{r^{2}(R, t)}{R^{2}}-q \frac{\partial}{\partial R}\left[\frac{r^{2}(R, t)}{R^{2}}\right] \\
& +\frac{\partial}{\partial R} \mu\left\{\left[\rho+\chi_{\infty} \frac{t_{\mathrm{B}}}{2-\alpha_{\mathrm{B}}} \phi_{\mathrm{B}}(R, 0, t)^{\left(2-\alpha_{\mathrm{B}}\right)}\right] \frac{R^{2}}{r^{2}(R, t)}\right. \\
+ & \left.\mu \int_{0}^{t} \chi\left(R, t^{\prime}\right) \phi_{\mathrm{B}}\left(R, t^{\prime}, t\right) \frac{r^{4}\left(R, t^{\prime}\right)}{r^{2}(R, t) R^{2}} \mathrm{~d} t^{\prime}\right\} \\
& -\frac{2}{R} q\left[\frac{r^{2}(R, t)}{R^{2}}-\frac{R}{r(R, t)}\right] \\
& +\frac{2}{R} \mu\left[\rho+\chi \frac{t_{\mathrm{B}}}{2-\alpha_{\mathrm{B}}} \phi_{\mathrm{B}}(R, 0, t)^{\left(2-\alpha_{\mathrm{B}}\right)}\right] \\
& \times\left[\frac{R^{2}}{r^{2}(R, t)}-\frac{r(R, t)}{R}\right] \\
& +\frac{2}{R} \mu \int_{0}^{t} \chi\left(R, t^{\prime}\right) \phi_{\mathrm{B}}\left(R, t^{\prime}, t\right)\left[\frac{r^{4}\left(R, t^{\prime}\right)}{r^{2}(R, t) R^{2}}-\frac{r(R, t) R}{r^{2}\left(R, t^{\prime}\right)}\right] \mathrm{d} t^{\prime} \\
& -\frac{2}{R} \mu \int_{0}^{t} \chi\left(R, t^{\prime}\right) \phi_{\mathrm{B}}\left(R, t^{\prime}, t\right) \frac{r(R, t) R}{r^{2}\left(R, t^{\prime}\right)} \mathrm{d} t^{\prime} \\
& \Rightarrow \frac{\partial q}{\partial R} \frac{r^{2}(R, t)}{R^{2}}=\frac{\partial f(R, t)}{\partial R}+\frac{2}{R} f(R, t) \\
& (\mathrm{A} 16 \mathrm{r}) \\
& \\
& \\
&
\end{aligned}
$$

$$
\begin{aligned}
& n(R, t)=\chi_{\infty} \frac{t_{\mathrm{B}}}{2-\alpha_{\mathrm{B}}}\left[\phi_{\mathrm{B}}(R, 0, t)\right]^{\left(2-\alpha_{\mathrm{B}}\right)} \\
& f(R, t)=\mu[\rho+n(R, t)] \frac{R^{2}}{r^{2}(R, t)} \\
& +\mu \int_{0}^{t} \chi\left(R, t^{\prime}\right) \phi_{\mathrm{B}}\left(R, t^{\prime}, t\right) \frac{r^{4}\left(R, t^{\prime}\right)}{r^{2}(R, t) R^{2}} \mathrm{~d} t^{\prime}
\end{aligned}
$$

To integrate for $q$, we rewrite (A16a)

$$
\begin{aligned}
& \frac{\partial q}{\partial R}=\frac{R^{2}}{r^{2}(R, t)} \frac{\partial f(R, t)}{\partial R}+\frac{2 R}{r^{2}(R, t)} f(R, t) \\
& -\frac{2 R}{r^{2}(R, t)} \mu[\rho+n(R, t)] \frac{r(R, t)}{R} \\
& -\frac{2 R}{r^{2}(R, t)} \mu \int_{0}^{t} \chi\left(R, t^{\prime}\right) \phi_{\mathrm{B}}\left(R, t^{\prime}, t\right) \frac{r(R, t) R}{r^{2}\left(R, t^{\prime}\right)} \mathrm{d} t^{\prime} \\
& =\frac{\partial}{\partial R}\left[\frac{R^{2}}{r^{2}(R, t)} f(R, t)\right] \\
& +\frac{2 R^{4}}{r^{5}(R, t)} f(R, t)-\mu[\rho+n(R, t)] \frac{2}{r(R, t)} \\
& -\mu \int_{0}^{t} \chi\left(R, t^{\prime}\right) \phi_{\mathrm{B}}\left(R, t^{\prime}, t\right) \frac{2 R^{2}}{r^{2}\left(R, t^{\prime}\right) r(R, t)} \mathrm{d} t^{\prime} \\
& =\frac{\partial}{\partial R}\left[\frac{R^{2}}{r^{2}(R, t)} f(R, t)\right]+\mu[\rho+n(R, t)] \frac{2}{r(R, t)}\left[\frac{R^{6}}{r^{6}(R, t)}-1\right] \\
& +\mu \int_{0}^{t} \chi\left(R, t^{\prime}\right) \phi_{\mathrm{B}}\left(R, t^{\prime}, t\right) \frac{2 R^{2}}{r^{2}\left(R, t^{\prime}\right) r(R, t)}\left[\frac{r^{6}\left(R, t^{\prime}\right)}{r^{6}(R, t)}-1\right] \mathrm{d} t^{\prime} \\
& \Rightarrow q=q_{0}(t)+\mu[\rho+n(R, t)] \frac{R^{4}}{r^{4}(R, t)} \\
& +\mu \int_{0}^{t} \chi\left(R, t^{\prime}\right) \phi_{\mathrm{B}}\left(R, t^{\prime}, t\right) \frac{r^{4}\left(R, t^{\prime}\right)}{r^{4}(R, t)} \mathrm{d} t^{\prime} \\
& +\mu \int_{A}^{R}\left\{\left[\rho+n\left(R^{\prime}, t\right)\right] \frac{2}{r\left(R^{\prime}, t\right)}\left[\frac{R^{\prime 6}}{r^{6}\left(R^{\prime}, t\right)}-1\right]\right\} \mathrm{d} R^{\prime} \\
& +\mu \int_{A}^{R}\left\{\int_{0}^{t} \chi\left(R^{\prime}, t^{\prime}\right) \phi_{\mathrm{B}}\left(R^{\prime}, t^{\prime}, t\right) \frac{2 R^{\prime 2}}{r^{2}\left(R^{\prime}, t^{\prime}\right) r\left(R^{\prime}, t\right)}\left[\frac{r^{6}\left(R^{\prime}, t^{\prime}\right)}{r^{6}\left(R^{\prime}, t\right)}-1\right] \mathrm{d} t^{\prime}\right\} \mathrm{d} R^{\prime}
\end{aligned}
$$

Combining eqn (A15a) and (A17) and using $\boldsymbol{\sigma}=\mathbf{P F}^{T}$, the true stress in the radial direction is

$$
\begin{aligned}
& \sigma_{r r}=-q_{0}(t) \\
& +\mu \int_{A}^{R}\left\{\left[\rho+n\left(R^{\prime}, t\right)\right] \frac{2}{r\left(R^{\prime}, t\right)}\left[1-\frac{R^{\prime 6}}{r^{6}\left(R^{\prime}, t\right)}\right]\right\} \mathrm{d} R^{\prime} \\
& +\mu \int_{A}^{R}\left\{\int_{0}^{t} \chi\left(R^{\prime}, t^{\prime}\right) \phi_{\mathrm{B}}\left(R^{\prime}, t^{\prime}, t\right) \frac{2 R^{\prime 2}}{r^{2}\left(R^{\prime}, t^{\prime}\right) r\left(R^{\prime}, t\right)}\left[1-\frac{r^{6}\left(R^{\prime}, t^{\prime}\right)}{r^{6}\left(R^{\prime}, t\right)}\right] \mathrm{d} t^{\prime}\right\} \mathrm{d} R^{\prime}
\end{aligned}
$$


The unknown $q_{0}(t)$ in (A18) is determined by the far field boundary condition

$$
\begin{aligned}
& \left.\sigma_{r r}\right|_{R \rightarrow \infty}=0 \\
& \Rightarrow q_{0}=\mu \int_{A}^{\infty}\left\{\left[\rho+n\left(R^{\prime}, t\right)\right] \frac{2}{r\left(R^{\prime}, t\right)}\left[1-\frac{R^{\prime 6}}{r^{6}\left(R^{\prime}, t\right)}\right]\right\} \mathrm{d} R^{\prime} \\
& +\mu \int_{A}^{\infty}\left\{\int_{0}^{t} \chi\left(R^{\prime}, t^{\prime}\right) \phi_{\mathrm{B}}\left(R^{\prime}, t^{\prime}, t\right) \frac{2 R^{\prime 2}}{r^{2}\left(R^{\prime}, t^{\prime}\right) r\left(R^{\prime}, t\right)}\left[1-\frac{r^{6}\left(R^{\prime}, t^{\prime}\right)}{r^{6}\left(R^{\prime}, t\right)}\right] \mathrm{d} t^{\prime}\right\} \mathrm{d} R^{\prime}
\end{aligned}
$$

Substituting (A19) back into (A18), the true radial stress is:

$$
\begin{aligned}
& \sigma_{r r}=-\mu \int_{R}^{\infty}\left\{\left[\rho+n\left(R^{\prime}, t\right)\right] \frac{2}{r\left(R^{\prime}, t\right)}\left[1-\frac{R^{\prime 6}}{r^{6}\left(R^{\prime}, t\right)}\right]\right\} \mathrm{d} R^{\prime} \\
& -\mu \int_{R}^{\infty}\left\{\int_{0}^{t} \chi\left(R^{\prime}, t^{\prime}\right) \phi_{\mathrm{B}}\left(R^{\prime}, t^{\prime}, t\right) \frac{2 R^{\prime 2}}{r^{2}\left(R^{\prime}, t^{\prime}\right) r\left(R^{\prime}, t\right)}\left[1-\frac{r^{6}\left(R^{\prime}, t^{\prime}\right)}{r^{6}\left(R^{\prime}, t\right)}\right] \mathrm{d} t^{\prime}\right\} \mathrm{d} R^{\prime}
\end{aligned}
$$

The other true stress components are obtained using (A19) and (A15b),

$$
\begin{aligned}
\sigma_{\theta \theta} & =\sigma_{\phi \phi} \\
& =\sigma_{r r}+\mu[\rho+n(R, t)]\left[\frac{r^{2}(R, t)}{R^{2}}-\frac{R^{4}}{r^{4}(R, t)}\right] \\
& +\mu \int_{0}^{t} \chi\left(R, t^{\prime}\right) \phi_{\mathrm{B}}\left(R, t^{\prime}, t\right)\left[\frac{r^{2}(R, t)}{r^{2}\left(R, t^{\prime}\right)}-\frac{r^{4}\left(R, t^{\prime}\right)}{r^{4}(R, t)}\right] \mathrm{d} t^{\prime}
\end{aligned}
$$

The unknown deformed radius $a(t)$ is determined by the boundary condition

$$
\begin{aligned}
& \left.\sigma_{r r}\right|_{R=A}=-p \\
& \Rightarrow \frac{p}{\mu_{\mathrm{net}}}=\int_{A}^{\infty}\left\{\left[\rho+n\left(R^{\prime}, t\right)\right] \frac{2}{r\left(R^{\prime}, t\right)}\left[1-\frac{R^{\prime 6}}{r^{6}\left(R^{\prime}, t\right)}\right]\right\} \mathrm{d} R^{\prime} \\
& +\int_{A}^{\infty}\left\{\int_{0}^{t} \chi\left(R^{\prime}, t^{\prime}\right) \phi_{\mathrm{B}}\left(R^{\prime}, t^{\prime}, t\right) \frac{2 R^{\prime 2}}{r^{2}\left(R^{\prime}, t^{\prime}\right) r\left(R^{\prime}, t\right)}\left[1-\frac{r^{6}\left(R^{\prime}, t^{\prime}\right)}{r^{6}\left(R^{\prime}, t\right)}\right] \mathrm{d} t^{\prime}\right\} \mathrm{d} R^{\prime}
\end{aligned}
$$

$a(t)$ is implicit in eqn (A21) through $r(R, t)=\left[R^{3}+a^{3}(t)-A^{3}\right]^{\frac{1}{3}}$.

Note since the instantaneous modulus for the linear and nonlinear cases are the same, the instantaneous responses for both cases are identical. Hence eqn (A13) is valid. The equation to be solved numerically in the nonlinear case is eqn (A21), with $\phi_{\mathrm{B}}\left(R, t^{\prime}, t\right), H\left(R, t^{\prime}, s\right), n(R, t)$ given by eqn (A14a), (A14b) and (A16b), respectively. Eqn (A21) must be solved together with eqn (A3b) (with $\chi(R, t<0)=\chi_{\infty}$ ) to determine the unknown healing rate $\chi(R, t)$. The solution is obtained iteratively: we start from $t=0$ with $a_{0}$ determined by eqn (A13). For each time increment, we guess the next cavity radius $a(t+\Delta t)$ and use this guess to find the healing rate $\chi(R, t+\Delta t)$ from eqn (A3b) or more precisely,

$$
1-\rho=\int_{-\infty}^{t+\Delta t} \chi\left(R, t^{\prime}\right) \phi_{\mathrm{B}}\left(R, t^{\prime}, t+\Delta t\right) \mathrm{d} t^{\prime}+\chi(R, t+\Delta t) t_{\mathrm{H}}
$$

Using the guessed value $a(t+\Delta t)$ we evaluate $\phi_{\mathrm{B}}(R, \tau, t+\Delta t)$ using (A14b) and (A14a). The updated healing rate $\chi(R, t+\Delta t)$ can now be found using (A22). Once we know $a(t+\Delta t)$ and $\chi(R, t+\Delta t)$, we can evaluate the RHS of (A21) at $t+\Delta t$ and determine the residue (res) which is the difference between this value and $p / \mu_{\text {net }}$. If the residue is too large, we will iterate with an updated guess of $a(t+\Delta t)$ based on the residue (via a NewtonRaphson scheme). Specifically, we take the first guess, $a_{1}(t+\Delta t)$ to be $a(t)$, the 2nd guess to be $a_{2}(t+\Delta t)=2 a(t)-a(t-\Delta t)$. In general, $\quad a_{k}(t+\Delta t)=a_{k-1}(t+\Delta t)+\frac{\operatorname{res}_{k-1}}{\operatorname{res}_{k-2}-\operatorname{res}_{k-1}} a_{k-1}(t+\Delta t)$. We continue with this iteration scheme until the residue is smaller than $10^{-4} \mathrm{p} / \mu_{\text {net }}$.

\section{A4. Cavity growth for linear and nonlinear viscoelastic solids}

Fig. 11 shows the time evolution of the normalized cavity radius $\bar{a}(t)$. The cavity grows faster in a nonlinear viscoelastic solid (three colored lines) compared to the linear viscoelastic case (black symbol). The smaller the critical strain $J_{0}$, the faster the bonds breaks at a given strain, and the faster the cavity grows.

\section{A5. Estimate of cohesive stress for elastomers}

The areal density of chains crossing a surface, $\Sigma$, is roughly ${ }^{35}$

$$
\Sigma \approx \nu_{x} a \sqrt{N_{x}}
$$

where $a$ is the size of a monomer, $\nu_{x}$ is the density of cross-links and $N_{x}$ is the number of monomers in a chain. $\nu_{x}$ can be estimated from the small strain shear modulus, $\mu$ by $\mu=\nu_{x} k_{\mathrm{B}} T$ where $k_{\mathrm{B}}$ is the Boltzmann constant and $T$ the absolute

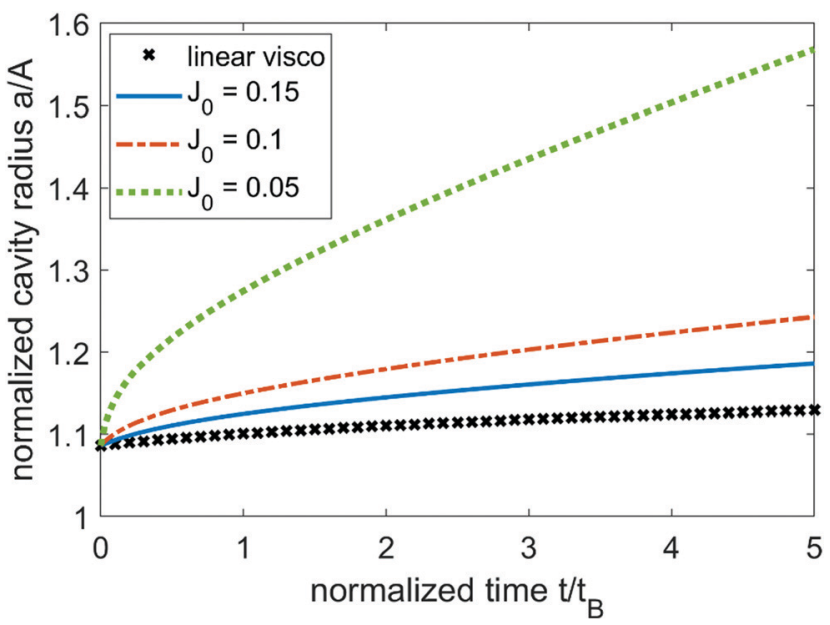

Fig. 11 Time evolution of the normalized cavity radius ( $A$ is the initial cavity radius and $a(t)$ is the current cavity radius). The black symbol is the linear viscoelastic case, whereas the three colored lines are the nonlinear viscoelastic cases with different $J_{0} s$. The cavity grows faster for the nonlinear viscoelastic solids. Smaller $J_{0}$ leads to faster cavity growth. 
temperature. The cohesive stress $\sigma_{\mathrm{c}}$ can be estimated using $\sigma_{\mathrm{c}} \approx \Sigma \times f_{\mathrm{c}-\mathrm{c}}$ where $f_{\mathrm{c}-\mathrm{c}} \approx 10^{-9}$ Newtons is roughly the force needed to break a carbon-carbon bond. Thus,

$$
\sigma_{\mathrm{c}} \approx \frac{\mu a}{k_{\mathrm{B}} T} f_{\mathrm{c}-\mathrm{c}} \sqrt{N_{x}}
$$

At room temperature $298 \mathrm{~K}, k_{\mathrm{B}} T \approx 4 \times 10^{-21} \mathrm{~J}$, the shear modulus of elastomers $\mu$ is roughly $0.5 \times 10^{6} \mathrm{~Pa}, a \approx 0.5 \times 10^{-9} \mathrm{~m}$ so

$$
\sigma_{\mathrm{c}} \approx \sqrt{N_{x}} 10^{8} \mathrm{~Pa}
$$

If we take $N_{x}=1000$, then $\sigma_{\mathrm{c}} \approx 3.5 \times 10^{9} \mathrm{~Pa}$.

\section{Acknowledgements}

C. Y. Hui, J. Guo and A. T. Zehnder acknowledges support by National Science Foundation, USA MoMS program under grant number 1903308. We thank the reviewers for their helpful comments and suggestions.

\section{References}

1 T. L. Smith, J. Polym. Sci., 1958, 32, 99-113.

2 A. N. Gent and S.-M. Lai, J. Polym. Sci., Part B: Polym. Phys., 1994, 32, 1543-1555.

3 J. Guo, R. Long, K. Mayumi and C.-Y. Hui, Macromolecules, 2016, 49, 3497-3507.

4 M. Liu, J. Guo, C.-Y. Hui, C. Creton, T. Narita and A. Zehnder, J. Rheol., 2018, 62, 991-1000.

5 W. G. Knauss, in Deformation and Fracture of High Polymers, ed. H. H. Kausch, J. A. Hassell and R. I. Jaffee, Springer US, Boston, MA, 1973, pp. 501-541.

6 R. A. Schapery, Int. J. Fract., 1975, 11, 141-159.

7 P. G. de Gennes, C. R. Acad. Sci. Paris, 1988, 1949-1953.

8 C. Hui, D. Xu and E. J. Kramer, J. Appl. Phys., 1992, 72, 3294-3304.

9 B. N. J. Persson and E. A. Brener, Phys. Rev. E: Stat., Nonlinear, Soft Matter Phys., 2005, 71, 036123.

10 T. D. Nguyen, H. Jerry Qi, F. Castro and K. N. Long, J. Mech. Phys. Solids, 2008, 56, 2792-2814.

11 W. G. Knauss, Int. J. Fract., 2015, 196, 99-146.

12 G. A. C. Graham and G. C. W. Sabin, Int. J. Eng. Sci., 1973, 11, 123-140.

13 J. R. Rice, Proceedings of the Eighth U.S. National Congress of Applied Mechanics, 1978, pp. 191-216.

14 L. N. McCartney, Int. J. Fract., 1980, 16, R229-R232.

15 R. M. Christensen, Int. J. Fract., 1980, 16, R233-R237.

16 H. K. Mueller and W. G. Knauss, J. Appl. Mech., 1971, 38, 483-488.

17 P. Rahulkumar, A. Jagota, S. J. Bennison and S. Saigal, Int. J. Solids Struct., 2000, 37, 1873-1897.

18 B. Moran and W. G. Knauss, J. Appl. Mech., 1992, 59, 95-101.

19 K. Mayumi, A. Marcellan, G. Ducouret, C. Creton and T. Narita, ACS Macro Lett., 2013, 2, 1065-1068.

20 K. J. Henderson, T. C. Zhou, K. J. Otim and K. R. Shull, Macromolecules, 2010, 43, 6193-6201.
21 W.-C. Lin, W. Fan, A. Marcellan, D. Hourdet and C. Creton, Macromolecules, 2010, 43, 2554-2563.

22 J.-Y. Sun, X. Zhao, W. R. K. Illeperuma, O. Chaudhuri, K. H. Oh, D. J. Mooney, J. J. Vlassak and Z. Suo, Nature, 2012, 489, 133-136.

23 T. L. Sun, T. Kurokawa, S. Kuroda, A. B. Ihsan, T. Akasaki, K. Sato, Md. A. Haque, T. Nakajima and J. P. Gong, Nat. Mater., 2013, 12, 932-937.

24 T. Narita, K. Mayumi, G. Ducouret and P. Hébraud, Macromolecules, 2013, 46, 4174-4183.

25 M. S. Green and A. V. Tobolsky, J. Chem. Phys., 1946, 14, 80-92.

26 F. Tanaka and S. F. Edwards, Macromolecules, 1992, 25, 1516-1523.

27 F. J. Vernerey, R. Long and R. Brighenti, J. Mech. Phys. Solids, 2017, 107, 1-20.

28 L. Leibler, M. Rubinstein and R. H. Colby, Macromolecules, 1991, 24, 4701-4707.

29 M. Rubinstein and A. N. Semenov, Macromolecules, 2001, 34, 1058-1068.

30 A. N. Semenov and M. Rubinstein, Macromolecules, 2002, 35, 4821-4837.

31 E. J. Regalado, J. Selb and F. Candau, Macromolecules, 1999, 32, 8580-8588.

32 P. Kujawa, A. Audibert-Hayet, J. Selb and F. Candau, J. Polym. Sci., Part B: Polym. Phys., 2004, 42, 1640-1655.

33 A. Tobolsky and H. Eyring, J. Chem. Phys., 1943, 11, 125-134.

34 S. Kundu and A. J. Crosby, Soft Matter, 2009, 5, 3963.

35 C. Creton and M. Ciccotti, Rep. Prog. Phys., 2016, 79, 046601.

36 R. M. Christensen, Theory of Viscoelasticity: An Introduction, Academic Press, New York, 2nd edn, 1982.

37 M. L. Williams, J. Appl. Mech., 1956, 109-114.

38 P. S. Steif, J. Appl. Mech., 1987, 54, 87-92.

39 J. R. Rice, Mathematical Fundamentals, Fracture: An Advanced Treatise, Academic Press, New York, 1968, vol. 2, pp. 191-311.

40 C. O. Horgan and D. A. Polignone, Appl. Mech. Rev., 1995, 48, 471-485.

41 L. Long and Z. Li, Int. J. Fract., 2018, 214, 105-113.

42 D. H. Kaelble, Trans. Soc. Rheol., 1971, 15, 275-296.

43 T. Cohen and A. Molinari, Int. J. Solids Struct., 2015, 69-70, 544-552.

44 R. Long, K. Mayumi, C. Creton, T. Narita and C.-Y. Hui, Macromolecules, 2014, 47, 7243-7250.

45 R. Long, K. Mayumi, C. Creton, T. Narita and C.-Y. Hui, J. Rheol., 2015, 59, 643-665.

46 A. E. Green and W. Zerna, Theoretical Elasticity, Oxford University Press, Oxford, 1954.

47 A. N. Gent and P. B. Lindley, Proc. R. Soc. London, Ser. A, 1959, 249, 195-205.

48 S. P. Timoshenko and J. N. Goodier, Theory of Elasticity, Mcgraw Hill, New York, 3rd edn, 1970.

49 D. H. Kaelble, Trans. Soc. Rheol., 1960, 4, 45-73.

50 C.-Y. Hui, Z. Liu, H. Minsky, C. Creton and M. Ciccotti, Soft Matter, 2018, 14, 9681-9692.

51 J. P. Gong, Y. Katsuyama, T. Kurokawa and Y. Osada, Adv. Mater., 2003, 15, 1155-1158. 
52 H. R. Brown, Macromolecules, 2007, 40, 3815-3818.

53 T. Matsuda, T. Nakajima, Y. Fukuda, W. Hong, T. Sakai, T. Kurokawa, U. Chung and J. P. Gong, Macromolecules, 2016, 49, 1865-1872.

54 A. Bellamine, E. Degrandi, M. Gerst, R. Stark, C. Beyers and C. Creton, Macromol. Mater. Eng., 2011, 296, 31-41.
55 P. Elzière, P. Fourton, Q. Demassieux, A. Chennevière, C. Dalle-Ferrier, C. Creton, M. Ciccotti and E. Barthel, Macromolecules, 2019, 52, 7821-7830.

56 E. Evans and K. Ritchie, Biophys. J., 1997, 72, 1541-1555.

57 R. Merkel, P. Nassoy, A. Leung, K. Ritchie and E. Evans, Nature, 1999, 397, 50-53. 